\title{
Studies of a full-scale mechanical prototype line for the ANTARES neutrino telescope and tests of a prototype instrument for deep-sea acoustic measurements
}

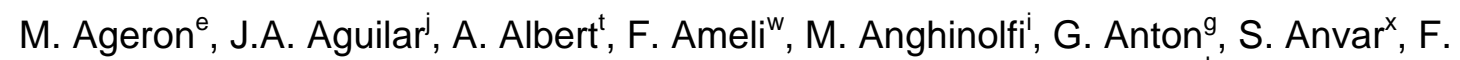
Ardellier-Desages $^{\mathrm{x}}$, E. Aslanides ${ }^{\mathrm{e}}$, J.-J. Aubert $^{\mathrm{e}}$, R. Auer $^{\mathrm{g}}, \mathrm{E}$. Barbarito ${ }^{\mathrm{b}}, \mathrm{S}$. Basa ${ }^{\mathrm{r}}, \mathrm{M}$. Battaglieri', M. Bazzotti ${ }^{\mathrm{C}}, \mathrm{Y}$. Becherini ${ }^{\mathrm{c}}$, N. Béthoux ${ }^{\mathrm{h}}$, J. Beltramelli ${ }^{\mathrm{x}}, \mathrm{V}$. Bertin ${ }^{\mathrm{e}}, \mathrm{A}$. Bigi ${ }^{\mathrm{v}}, \mathrm{M}$. Billault $^{\mathrm{e}}$, R. Blaes ${ }^{\mathrm{t}}$, N. de Botton ${ }^{\mathrm{x}}$, M.C. Bouwhuis ${ }^{\mathrm{u}}, \mathrm{R}$. Bruijn ${ }^{\mathrm{u}}, \mathrm{z}, \mathrm{J}$. Brunner ${ }^{\mathrm{e}}$, G.F. Burgio ${ }^{\dagger}, \mathrm{J}$.

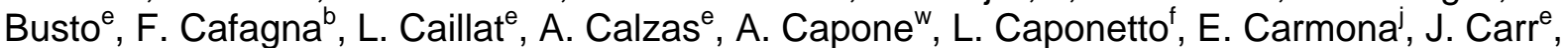
D. Castel ${ }^{t}$, E. Castorinav, V. Cavasinni ${ }^{v}$, S. Cecchini ${ }^{c}$, A. Ceres ${ }^{b}$, P. Charvis ${ }^{h}$, P. Chauchot ${ }^{k}, T$. Chiarusiw $^{w}$, M. Circella ${ }^{b}$, J.-Y. Coail ${ }^{k}$, C. Colnard ${ }^{u}$, C. Compére ${ }^{k}$, R. Coniglione ${ }^{s}$, N. Cottini ${ }^{v}$, P. Coyle $^{\mathrm{e}}$, S. Cuneo', A.-S. Cussatlegras ${ }^{\mathrm{d}}$, G. Damy ${ }^{\mathrm{k}}$, R. van Dantzigu, G. DeBonis ${ }^{\mathrm{w}}$, C. De Marzo $^{b}$, R. De Vita', I. Dekeyser ${ }^{d}$, E. Delagnes ${ }^{x}$, D. Denans ${ }^{x}$, A. Deschamps ${ }^{h}$, J.-X. Dessa ${ }^{h}$, J.-J. Destelle ${ }^{\mathrm{e}}$, B. Dinkespieler ${ }^{\mathrm{e}}$, C. Distefanos ${ }^{\mathrm{s}}$, C. Donzaud ${ }^{\mathrm{x}}, 3, \mathrm{~J}-\mathrm{F}$. Drogou', F. Druillole ${ }^{\mathrm{x}}, \mathrm{D}$. Durand $^{\mathrm{x}}$, J.-P. Ernenwein ${ }^{\mathrm{t}}$ S. Escoffier ${ }^{\mathrm{e}}$, E. Falchini ${ }^{\mathrm{v}}$, S. Favard ${ }^{\mathrm{e}}$, F. Fehr ${ }^{\mathrm{g}}$, F. Feinstein ${ }^{\mathrm{e}}$, C.

Fiorello $^{b}$, V. Flaminio ${ }^{v}$, K. Fratini', J.-L. Fuda ${ }^{d}$, S. Galeotti ${ }^{\mathrm{v}}$, J.-M. Gallone ${ }^{\mathrm{n}}$, G. Giacomellic, N.

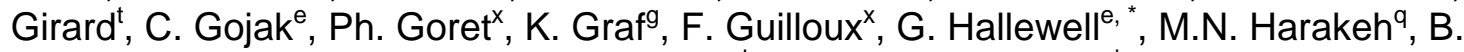
Hartmann $^{g}$, A. Heijboer ${ }^{u}$, z, E. Heine ${ }^{u}, Y$. Hello ${ }^{h}$, J.J. Hernández-Rey ${ }^{j}$, J. Hößl ${ }^{g}$, C. Hoffman ${ }^{n}$, J. Hogenbirk ${ }^{\mathrm{u}}$, J.R. Hubbard ${ }^{\mathrm{x}}, \mathrm{M}$. Jaquet ${ }^{\mathrm{e}}, \mathrm{M}$. Jaspers $^{\mathrm{u}}, \mathrm{z}, \mathrm{M}$. de Jong ${ }^{\mathrm{u}}$, F. Jouvenot ${ }^{\mathrm{x}}, \mathrm{N}$. Kalantar-Nayestanaki ${ }^{q}$, A. Kappes ${ }^{g}$, T. Karg ${ }^{g}$, U. Katz ${ }^{g}$, P. Keller ${ }^{\mathrm{e}}$, J.P. Kneib ${ }^{\mathrm{r}}$, E. Kok ${ }^{\mathrm{u}}, \mathrm{H}$. Kok $^{u}$, P. Kooijman ${ }^{u}$, y, C. Kopper ${ }^{g}$, A. Kouchner ${ }^{a}$, W. Kretschmer ${ }^{g}$, A. Kruijer ${ }^{u}$, S. Kuch ${ }^{g}$, P.

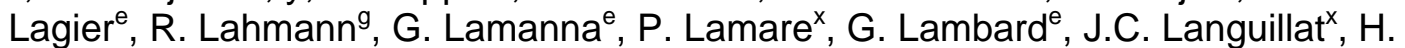
Laschinsky $^{g}$, J. Lavalle ${ }^{e}, Y$. Le Guen ${ }^{k}, H$. Le Provost ${ }^{x}, A$. Le Van Suu ${ }^{e}$, D. Lefévre ${ }^{d}, T$.

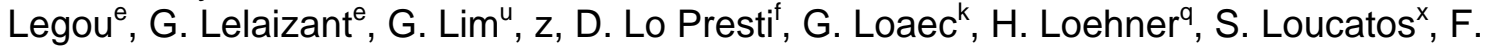
Louis $^{\mathrm{x}}$, F. Lucarelliw ${ }^{\mathrm{w}}$, V. Lyashuk ${ }^{\mathrm{p}}, \mathrm{S}$. Mangano ${ }^{\mathrm{u}}$, M. Marcelin ${ }^{\mathrm{r}}$, A. Margiotta ${ }^{\mathrm{c}}$, R. Masullow $^{\mathrm{w}}, \mathrm{F}$. Mazéas $^{k}$, A. Mazure ${ }^{r}$, R. Megna ${ }^{b}$, M. Melissas ${ }^{e}, E$. Migneco $^{s}, M$. Mongelli ${ }^{b}$, T. Montaruli ${ }^{b}, M$. Morganti $^{\mathrm{V}}$, L. Moscoso ${ }^{\mathrm{x}}, \mathrm{H}$. Motz ${ }^{\mathrm{g}}$, M. Musumeci ${ }^{\mathrm{s}}, \mathrm{C}$. Naumann ${ }^{\mathrm{g}}$, M. Naumann-Godo ${ }^{g}, \mathrm{~V}$.

Niess $^{\mathrm{e}}$, A. Noble ${ }^{\mathrm{e}}$, C. Olivetto ${ }^{n}$, R. Ostasch ${ }^{\mathrm{g}}$, N. Palanque-Delabrouille ${ }^{\mathrm{x}}$, P. Payre, H.Z. Peek $^{v}$, A. Perez ${ }^{j}$, C. Petta ${ }^{\dagger}$, P. Piattelli ${ }^{\mathrm{s}}$, R. Pillet ${ }^{h}$, J.-P. Pineau ${ }^{n}$, J. Poinsignon ${ }^{x}$, V. Popa ${ }^{\circ}$, T. Pradier $^{n}$, C. Racca ${ }^{n}$, N. Randazzo ${ }^{f}$, J. van Randwijk ${ }^{u}$, D. Real ${ }^{j}$, M. Regnier ${ }^{h}$, B. van Rens ${ }^{u}, F$. Réthorée, P. Rewiersma", G. Riccobenes, V. Rigaud', M. Ripani', V. Roca', C. Rodav, J.F.

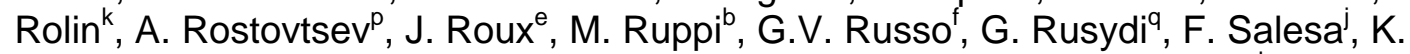

Salomon $^{g}$, P. Sapienza ${ }^{\mathrm{s}}$, F. Schmitt ${ }^{\mathrm{g}}$, J.-P. Schuller ${ }^{\mathrm{x}}$, R. Shanidze $^{\mathrm{g}}$, I. Sokalski ${ }^{\mathrm{b}}$, T. Spona ${ }^{g}$, M. Spurio ${ }^{c}$, G. van der Steenhoven ${ }^{u}$, T. Stolarczyk ${ }^{\mathrm{x}}$, K. Streeb ${ }^{g}$, L. Sulak ${ }^{\mathrm{e}}$, M. Taiuti, C. Tamburini $^{d}$, C. Tao ${ }^{\mathrm{e}}$, L. Tasca $^{\mathrm{r}}, \mathrm{G}$. Terreni ${ }^{\mathrm{v}}$, F. Urbano', P. Valdy', V. Valente ${ }^{\mathrm{w}}$, B. Vallage ${ }^{\mathrm{x}}, \mathrm{G}$.

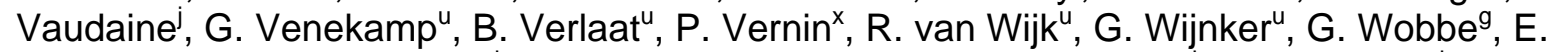
de Wolf ${ }^{u}$, z, A.-F. Yao ${ }^{d}$, D. Zaborov ${ }^{p}$, H. Zaccone ${ }^{x}$, J.D. Zornoza ${ }^{j}$ and J. Zúñiga ${ }^{j}$

\footnotetext{
${ }^{a}$ APC_-AstroParticule et Cosmologie, UMR 7164 (CNRS, Université Paris 7, CEA, Observatoire de Paris), 10 rue Alice Domon et Léonie Duquet, 75205 Paris Cedex 13, France

${ }^{b}$ Dipartimento Interateneo di Fisica e Sezione INFN, Via E. Orabona 4, 70126 Bari, Italy

'Dipartimento di Fisica dell'Università e Sezione INFN, Viale Berti Pichat 6/2, 40127 Bologna, Italy

${ }^{d}$ COM-Centre d'Océanologie de Marseille, CNRS/INSU et Université de la Méditerranée, 163 Avenue de Luminy, Case 901, 13288 Marseille Cedex 9, France

${ }^{e}$ CPPM-Centre de Physique des Particules de Marseille, CNRS/IN2P3 et Université de la Méditerranée, 163 Avenue de Luminy, Case 902, 13288 Marseille Cedex 9, France
} 
fDipartimento di Fisica ed Astronomia dell'Università e Sezione INFN, Viale Andrea Doria 6, 95125 Catania, Italy ${ }^{9}$ Friedrich-Alexander-Universität Erlangen-Nürnberg, Physikalisches Institut, Erwin-Rommel-Str. 1, D-91058 Erlangen, Germany

${ }^{\mathrm{h}}$ GéoSciences Azur, CNRS/INSU, IRD, Université de Nice Sophia-Antipolis, Université Pierre et Marie CurieObservatoire Océanologique de Villefranche, BP48, 2 quai de la Darse, 06235 Villefranche-sur-Mer Cedex, France

'Dipartimento di Fisica dell'Università e Sezione INFN, Via Dodecaneso 33, 16146 Genova, Italy ${ }^{\mathrm{j}}$ IFIC—Instituto de Física Corpuscular, Edificios Investigación de Paterna, CSIC—Universitat de València, Apdo. de Correos 22085, 46071 Valencia, Spain

kIFREMER-Centre de Brest, BP 70, 29280 Plouzané, France

IIFREMER-Centre de Toulon/La Seyne Sur Mer, Port Brégaillon, Chemin Jean-Marie Fritz, 83500, La Seyne sur Mer, France

mINAF-IASF, via P. Gobetti 101, 40129 Bologna, Italy

nIPHC-Institut Pluridisciplinaire Hubert Curien, Université Louis Pasteur (Strasbourg 1) et IN2P3/CNRS, 23 rue du Loess, BP 28, 67037 Strasbourg Cedex 2, France

${ }^{\circ}$ Institute for Space Sciences, 77125 Bucharest, Magurele, Romania

PITEP_Institute for Theoretical and Experimental Physics, B. Cheremushkinskaya 25, 117259 Moscow, Russia

${ }^{q}$ Kernfysisch Versneller Instituut (KVI), University of Groningen, Zernikelaan 25, 9747 AA Groningen, The

Netherlands

'LAM - Laboratoire d'Astrophysique de Marseille, CNRS/INSU et Université de Provence, Traverse du SiphonLes Trois Lucs, BP 8, 13012 Marseille Cedex 12, France

SINFN_Labaratori Nazionali del Sud (LNS), Via S. Sofia 44, 95123 Catania, Italy

tGRPHE-Groupe de Recherche en Physique des Hautes Energies, Université de Haute Alsace, 61 Rue Albert Camus, 68093 Mulhouse Cedex, France

uNationaal Instituut voor Kernfysica en Hoge-Energiefysica (NIKHEF), Kruislaan 409, 1098 SJ Amsterdam, The Netherlands

v'Dipartimento di Fisica dell'Università e Sezione INFN, Largo B. Pontecorvo 3, 56127 Pisa, Italy

"Dipartimento di Fisica dell'Università "La Sapienza" e Sezione INFN, P.le Aldo Moro 2, 00185 Roma, Italy

${ }^{x}$ DSM/DAPNIA-Direction des Sciences de la Matière, Laboratoire de Recherche sur les lois Fondamentales de I'Univers, CEA Saclay, 91191 Gif-sur-Yvette Cedex, France

y Universiteit Utrecht, Faculteit Betawetenschappen, Princetonplein 5, 3584 CC Utrecht, The Netherlands

${ }^{z}$ Universiteit van Amsterdam, Instituut voor Hoge-Energiefysica, Kruislaan 409, 1098 SJ Amsterdam, The Netherlands.

*: Corresponding author : G. Hallewell, email : gregh@cppm.in2p3.fr

\begin{abstract}
:
A full-scale mechanical prototype line was deployed to a depth of $2500 \mathrm{~m}$ to test the leak tightness of the electronics containers and the pressure-resistant properties of an electromechanical cable under evaluation for use in the ANTARES deep-sea neutrino telescope. During a month-long immersion study, line parameter data were taken using miniature autonomous data loggers and shore-based optical time domain reflectometry. Details of the mechanical prototype line, the electromechanical cable and data acquisition are presented. Data taken during the immersion study revealed deficiencies in the pressure resistance of the electromechanical cable terminations at the entry points to the electronics containers. The improvements to the termination, which have been integrated into subsequent detection lines, are discussed. The line also allowed deep-sea acoustic measurements with a prototype hydrophone system. The technical setup of this system is described, and the first results of the data analysis are presented.
\end{abstract}

Keywords: ANTARES; Underwater neutrino telescope; Leak detection; Optical time domain reflectometry; Electromechanical cable; Sea deployment 


\section{Introduction}

The ANTARES undersea neutrino telescope [1, 2] - fig. 1 - will contain twelve detection lines at a depth of $2500 \mathrm{~m}$ in the Mediterranean Sea near Toulon. Each line rises $450 \mathrm{~m}$ from a seabed anchor and will be kept in tension by a submerged flotation buoy. The active length of each line comprises 75 optical modules [3] housing photomultipliers [4] inside glass pressure spheres at 25 'storeys' spaced at vertical intervals of 14.5 $\mathrm{m}$ starting at an altitude of $\sim 100 \mathrm{~m}$ above the seabed. Each storey is composed of a tubular titanium support frame - fig. 2 - with attachment points for optical modules and a central electronics container. The frame also contains upper and lower mechanical anchorages for the vertical electro-mechanical cable ${ }^{1}$ (EMC) which links the electric power and fibre-optic data-flow services between successive storeys and contains the braided web of $\operatorname{Kevlar}{ }^{\circledR}$ aramid fibres which gives the line its mechanical strength.

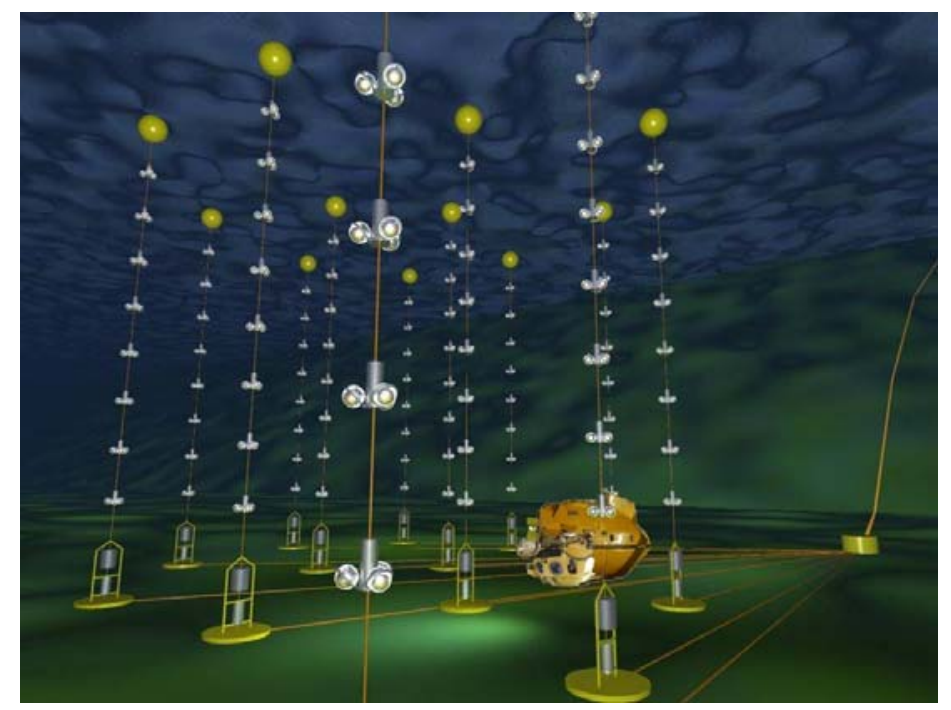

Fig. 1. Artist's view of the ANTARES underwater neutrino telescope.

Detection lines in the telescope are free to swing and rotate in the undersea currents at the ANTARES site. These have typical values (based on 21/2 years' continuous data taking with a Doppler current profiler) of $4-10 \mathrm{cms}^{-1}\left(0.14-0.36 \mathrm{~km} . \mathrm{hr}^{-1}\right)$, with rare peaks as high as $30 \mathrm{cms}^{-1}\left(\sim 1.1 \mathrm{~km} . \mathrm{hr}^{-1}\right)$. Since the specification on pointing precision of the telescope requires that optical module positions are known to a precision of around $20 \mathrm{~cm}$, each line is equipped with hydrophones at five different heights, whose signals (multiple time delay measurements) are used to triangulate the line in real time relative to a sea floor transponder net operating in the 5-60 kHz frequency band. The 'fine structure' (3D curvature, twist) of each line is given in more detail by combined electronic tiltmeter/compass instruments located in each of its 25 electronics containers. The acoustic positioning system, which will be the subject of a future publication, was used in this work for the testing of a prototype undersea acoustic detection module using a technology similar to that which might be used in a future very large volume underwater neutrino telescope.

The electronics in the container shown in fig. 2 operates in a dry air atmosphere at a pressure of 1 bar while the surrounding seawater is at 250 bar. The container is constructed entirely in titanium, based on a cylinder with outer (inner) diameter 18(15.8) cm, two $30 \mathrm{~mm}$ thick end flanges and three threaded rods which are tensioned to compress the flange O-ring seals during closure.

Electrical and fibre-optic services enter the container through the end flanges. The lower flange (fig. 3) is pierced with holes into which SubConn ${ }^{2}$ multi-conductor electrical connectors are inserted. These connectors are equipped with O-rings and are tightened to the specified torque.

\footnotetext{
1 Manufacturer: LAPP Muller SA, 83310 Grimaud, France

${ }^{2}$ MacArtney Underwater Technology group http://www.subconn.com
} 


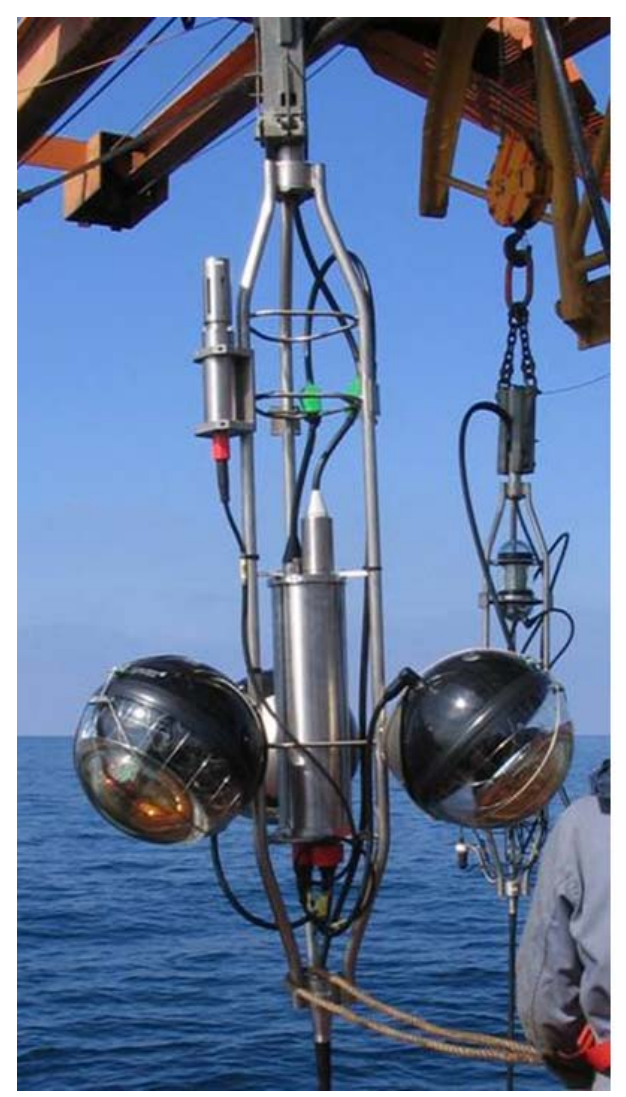

Fig. 2. View of an optical module support frame showing the mounting of the electronics cylinder together with the EMC penetrators and cable routing.

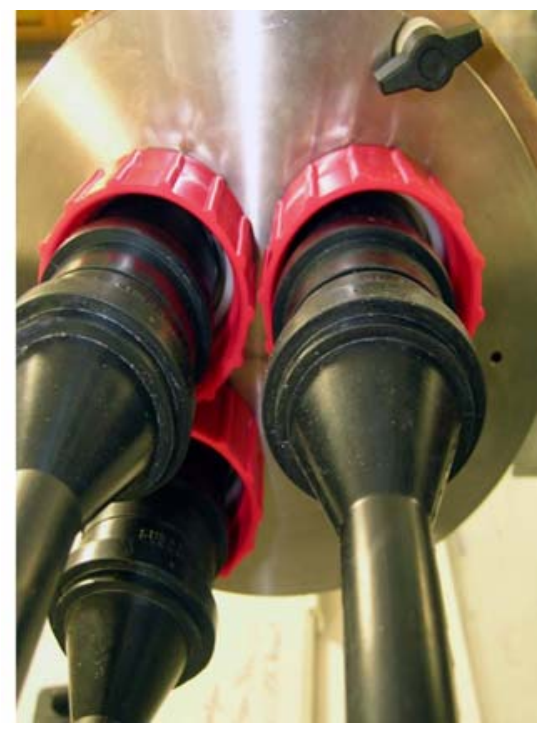

Fig. 3. View of the lower flange of an electronics container showing the three connections to the optical module spheres plugged into the SubConn ${ }^{\circledR}$ bulkhead connectors. 
Their leak tightness is verified in a pressure test with each flange mounted on a dummy cylinder inside a caisson pressurised to 310 bar for 1 hour $^{3}$. Most connector flanges are fitted with the three 12-pin connectors used for optical module connection; however in some storeys an additional connector is provided to service a hydrophone or an LED flasher beacon [5] for detector calibration.

The reliability of chaining the nine electric power conductors and 21 optical fibres contained in the EMC core is improved through the use of 'penetrators' - rather than connectors - at the entry points to each electronics container. Two penetrators are located on the upper flange and are each sealed to it with a pair of O-rings. Within each container, the conductors are soldered to power distribution boards and fibres are fusion spliced either to fibre pigtails directly attached to the laser driver and pin diode receivers of the electronics cards, or to fibres which continue the data path via the following EMC segment to the neighbouring container.

Figure 4 illustrates the cross section of the EMC non-mechanical core. Three fibre tubes and nine electrical conductors are helically wound during cable fabrication. The three stainless steel tubes of outer diameter $2 \mathrm{~mm}$ each contain seven optical fibres. ${ }^{4}$ The tubes are made from a $200 \mu \mathrm{m}$ thick foil formed around the fibres during the cable drawing process and closed longitudinally by electron beam welding. These tubes must resist the sea pressure of 250 bar while undergoing the repeated torsional rotations that the detection line will be subjected to in the undersea current. A previous development version of the EMC with plastic fibre tubes had been used to link the electronics containers in a 5-storey prototype line. The tubes in some cable segments collapsed under long-term deep-sea pressure, causing increased optical attenuation which prevented signal propagation through some fibres.

Since glass optical fibres are sensitive to hydrogen attack on contact with seawater, the fibre tubes contain a gel to inhibit capillary wicking through the fibre interstices, retarding the growth of the zone open to hydrogen corrosion should the tube become pierced. It should be noted that the stainless steel of the fibre tubes, unlike titanium, is not particularly resistant to sea water corrosion. Nor, in the event of a high pressure sea water leak through the polyethylene outer jacket, is the EMC core longitudinally waterproof. Additional blocking must be introduced to prevent high pressure water entering the electronics containers by longitudinal migration through the cable.

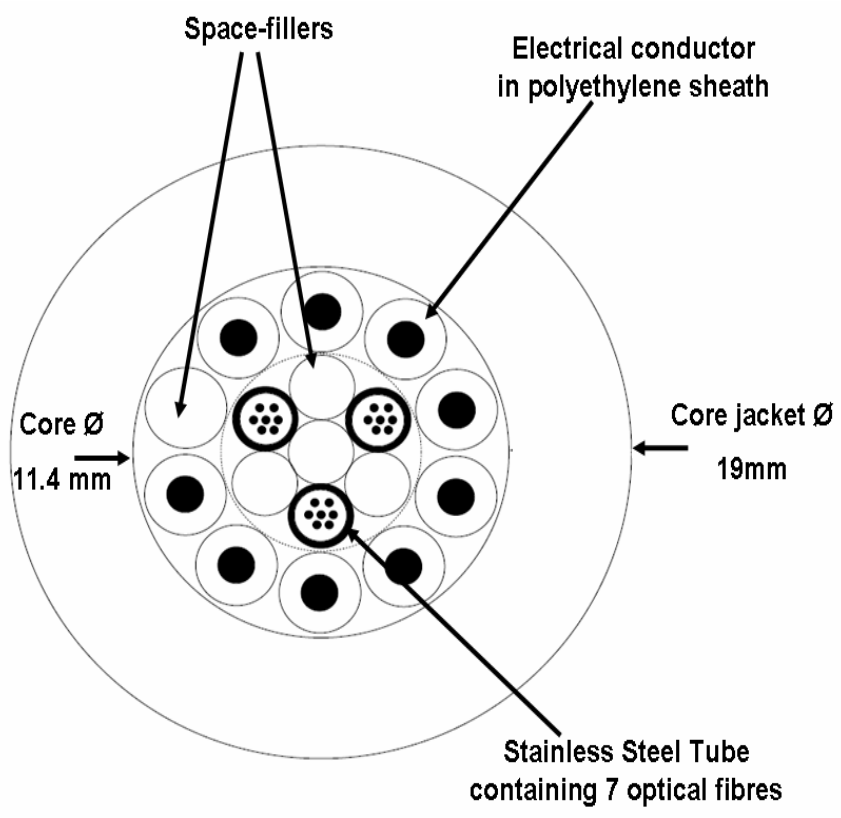

Fig. 4. Cross section of the EMC non-mechanical core.

\footnotetext{
${ }^{3}$ A test protocol at $120 \%$ service pressure recommended by IFREMER, the French oceanographic institute

${ }^{4}$ Corning SMF28 monomode optical fibre, n 1.47 @1550 nm.
} 
Two types of titanium shell penetrator are used for the EMC entry into the electronics containers. Figures 5a \& 5b illustrate a 'Water-Blocking' (WB) penetrator ${ }^{5}$, which is watertight against seawater penetration either into the fibre tubes or between the copper conductors and their sheathes. Although such infiltration is unlikely, being the result of a failure of both the EMC outer jacket and the optical fibre tube or insulation sheath, damage caused by snagging during deployment might provoke this type of leak.

In the central $12 \mathrm{~cm}$-long break-out tube of the WB penetrator (fig. 5a) each optical fibre and electrical conductor is separated and individually glued or pinned into a leak-tight feedthrough traversing a pressureresistant bulkhead. This bulkhead is the last line of leak defence of the electronics container and is illustrated on the right of fig. $5 \mathrm{a}$, and face-on in fig. 5b. The 21 optical fibres are glued into a $3 \mathrm{~mm}$ diameter stainless steel tube which is swaged into a bulkhead union ${ }^{6}$, while the electrical conductors are soldered to both ends of feedthrough pins insulated from the bulkhead with glass-to-metal bead seals.

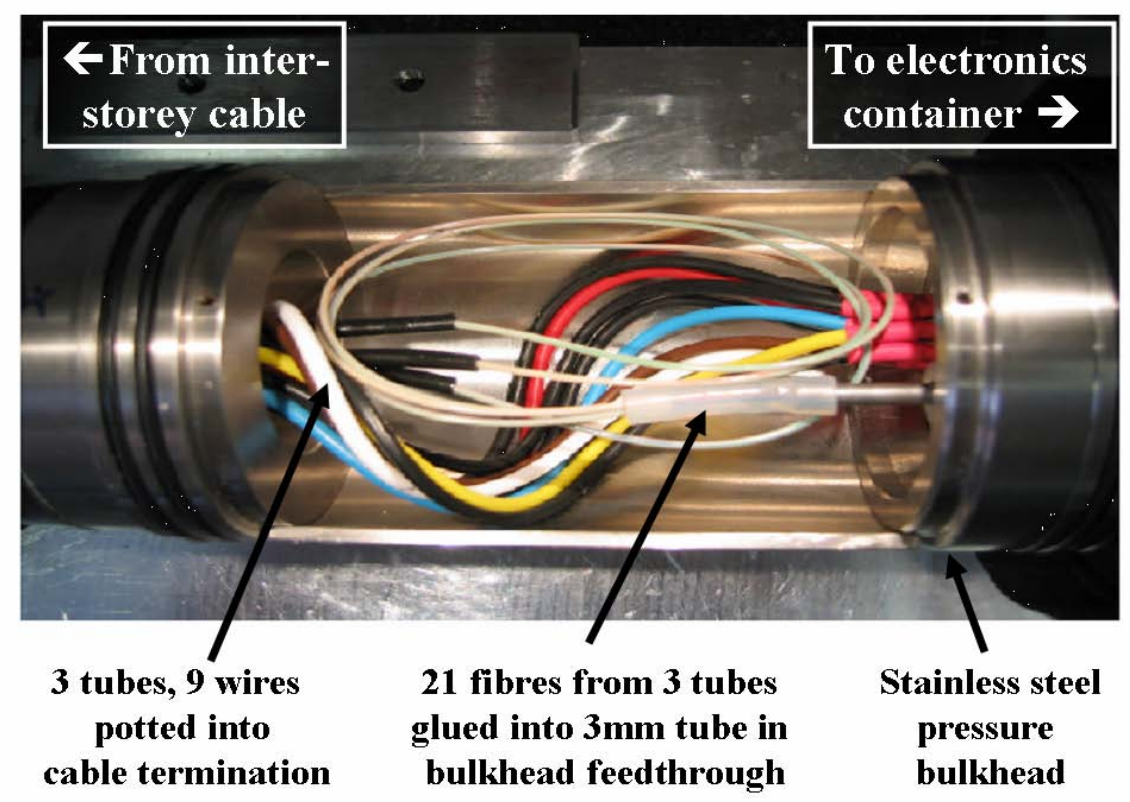

Fig. 5a. Internal detail of cable and fibre breakout in the central region of a "Water-Blocking" penetrator.

The water-side restraint of the EMC core is in the anchorage shown on the left of fig. 5a. The three fibre tubes, the outer polyethylene sheathes of the nine electrical conductors and the polyethylene jacket of the EMC core are retained in the inner bore of the penetrator by a two-part acrylic adhesive ${ }^{7}$ potting plug.

This anchorage is leak tight to water that has infiltrated the core interstices (fig. 4) between the fibre tubes and jackets of the electrical conductors, provided it has not also penetrated either into the fibre tube(s) or under the jacket(s) of the electrical conductor(s). Despite this limitation, this anchorage forms the basis of a simplified "Non-Water-Blocking" (NWB) penetrator ${ }^{8}$, which for financial reasons equips the majority $(80 \%)$ of the 300 inter-storey EMC sections in ANTARES. True water blocking penetrators - comprising all the components of fig. $5 \mathrm{a}, \mathrm{b}$ - protect the entry into the electronics container of every fifth storey. A single damaged EMC section or flooded container may propagate leaks into at most, one 'sector' of five contiguous electronics containers, out of the 25 in a detection line. The result of such a leak will be the blocking of data transmission and power delivery to all sectors above the flooded zone and corrosion damage to the electronics and the non-mechanical cores of the EMC segments linking the flooded storeys. The mechanical integrity of the detection line will, however, still be assured by the non-corrodible Kevlar webbing in the EMC external jacket.

\footnotetext{
${ }^{5}$ Manufactured \& fitted by MacArtney, 6710 Esbjerg V, Denmark \& SEDI, 91080 Courcouronnes, France

${ }^{6}$ Based on the principle of the 'Swagelok' range of tube fittings http://www.swagelok.com

${ }^{7}$ DP 8010 two-part adhesive by 3M Corporation

${ }^{8}$ Manufactured \& fitted by MacArtney, 6710 Esbjerg V, Denmark
} 


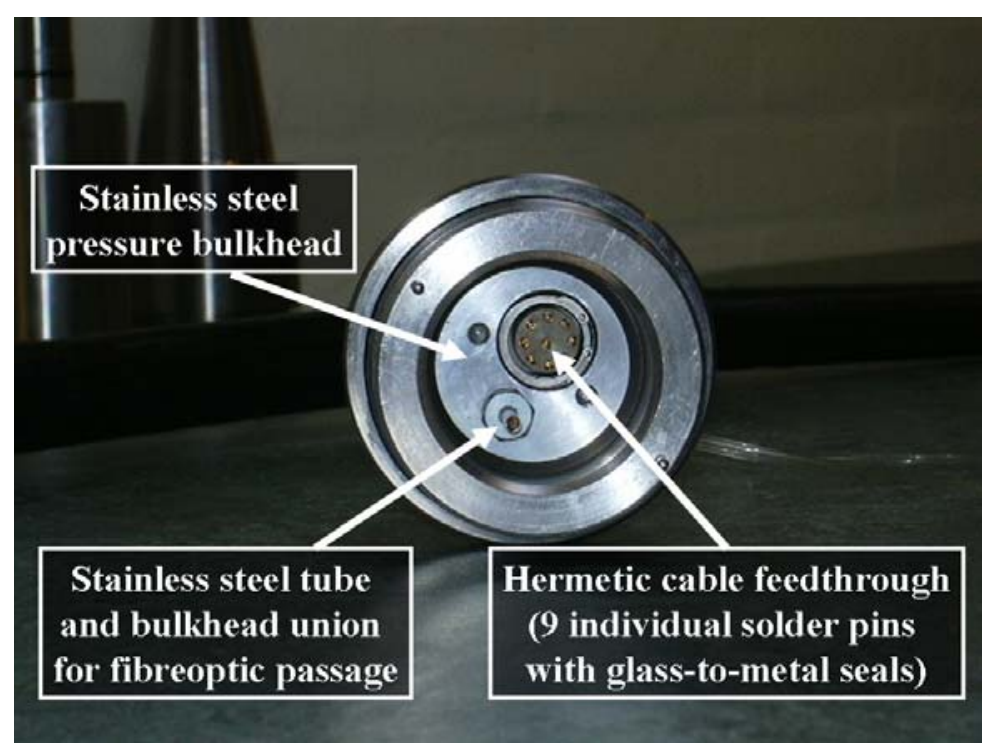

Fig. 5b. Hermetic feedthroughs for power conductors and optical fibres in a "Water-Blocking" penetrator.

\section{Aims of the full scale mechanical prototype line}

In a 2003 deployment study with a short prototype line of five storeys and a previous development version of the EMC leaks were seen into the electronics containers in two storeys. Optical transmission also became blocked in several fibres due the pressure collapse of plastic fibre tubes, as mentioned earlier.

A new full scale mechanical prototype line - fig. 6 - with 23 storeys was built and deployed in March 2005 to test a statistically-significant sample of electronics containers and sections of the new design of EMC.

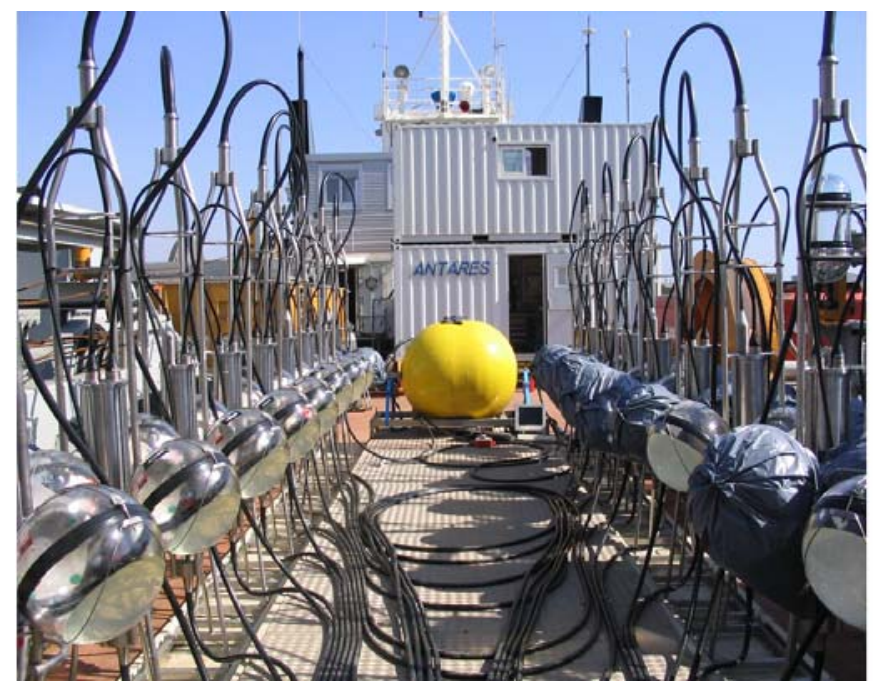

Fig. 6. The full scale mechanical prototype line on the deck of the deployment ship, showing the simulated optical modules, flotation buoy and the inter-storey sections of electro-mechanical cable

The new line was intended to simulate as many aspects of a real detector line as possible, including buoyancy. Weights of optical modules were simulated using pressure spheres containing a weight of silicone gel equivalent to that of a photomultiplier and other internal components in a real optical module. 
The line used a standard version of the ANTARES sea anchor [1], employing a detachable steel weight and an acoustic release mechanism. Reception of an encrypted sound pulse triggered the acoustic release, the weight reduction allowing the flotation buoy to raise the line and the recoverable portion of the anchor to the surface in $\sim 45$ minutes (sections 4,5 ).

Leak sensors were built into the electronics containers of each storey and read out by battery-powered autonomous data loggers (section 3). In addition, the optical attenuation in a looped pair of fibres in each of the three fibre tubes was monitored by a data logger connected to an optical attenuation monitor (section 3).

The prototype mechanical line was equipped with autonomous mechanical instrument packages incorporating 3-axis dynamometry and tiltmetry to record the mechanical history of the line during deployment and recovery (section 5). A prototype acoustic detection module (section 6) was also deployed with the aim of using part of the 5-60 kHz bandwidth of the ANTARES sea floor acoustic triangulation system to validate the acoustic data-taking setup at high sea pressure and help in the development of acoustic signal processing algorithms for use in a possible future very large volume $\left(>1 \mathrm{~km}^{3}\right)$ deep sea telescope for the detection of extraterrestrial neutrinos with energies exceeding $10^{17} \mathrm{eV}$.

\section{The autonomous data loggers}

TinyTag ${ }^{\circledR}$ battery-powered autonomous data loggers ${ }^{9}$ were used for the monitoring of 23 leak sensors and the three internal optical paths. Each TinyTag had a memory capacity of 16384 readings of a single sensor channel. Although existing in versions for direct monitoring of various sensor types, the TinyTags chosen for our application had a full-scale DC input range of $200 \mathrm{mV}$. Each TinyTag could be programmed for data taking period, interval between readings and immediate or delayed start through its RS232 port. The stored data were read out through RS232. A 15 minute interval between successive reads was selected to allow a maximum autonomy of 170 days.

\subsection{Leak Monitoring}

Figures 7a-c illustrate the installation of a leak-monitoring TinyTag. The leak sensor is based on a
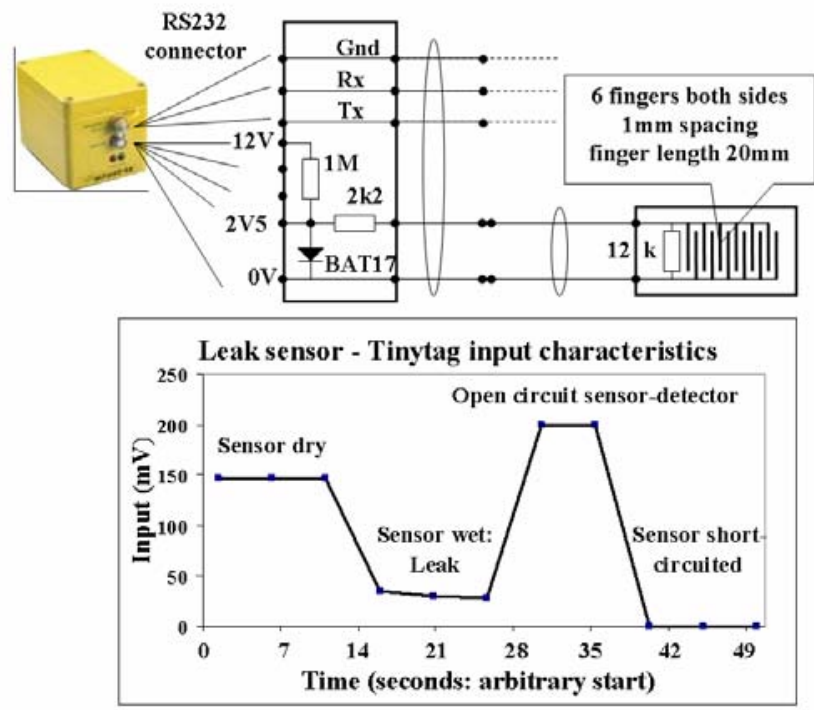

Fig. 7a. TinyTag connection to leak detector and voltage input to TinyTag under various sensor conditions.

small double-sided printed circuit with 12 gold plated fingers. When dry its characteristic impedance of 12 $\mathrm{k} \Omega$ gives a $150 \mathrm{mV}$ input to the TinyTag. When short-circuited by water, this drops to around $30 \mathrm{mV}$ (fig. 7a). The leak sensor is placed at the lowest part of the electrical container, attached to the optical module connector flange (fig 7b). To avoid damage to the TinyTags by leaks into the containers under test, they are fitted in optical module spheres whose leak tightness - based on earlier experience - was not in question,

\footnotetext{
${ }^{9}$ Mfr: Gemini Data Loggers Ltd, Chichester PO19 8UJ, UK
} 
and held in position using silicone gel (fig. 7c). Sensor connection to the TinyTag uses an optical module cable plugged into one of the SubConn connectors, while the RS232 access for programming and data collection passes through a second connector.

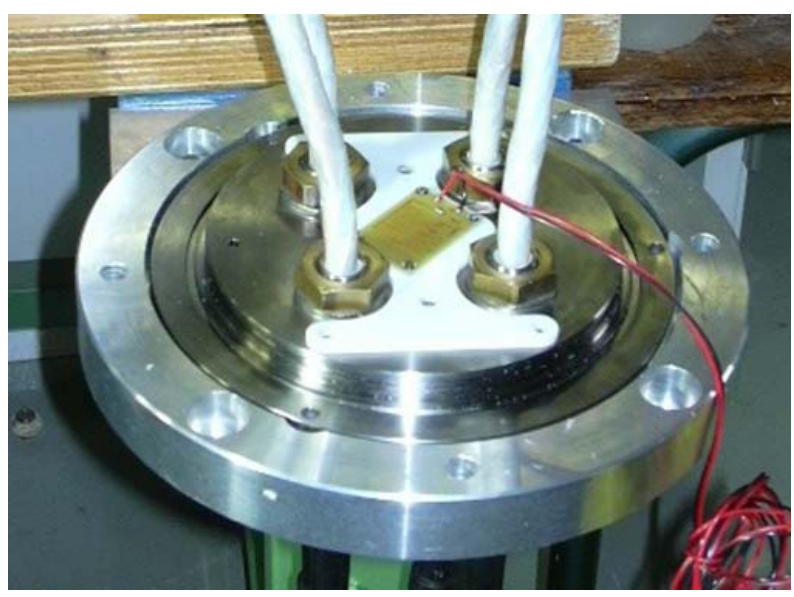

Fig.7b. Leak sensor installation on the optical module connector flange.

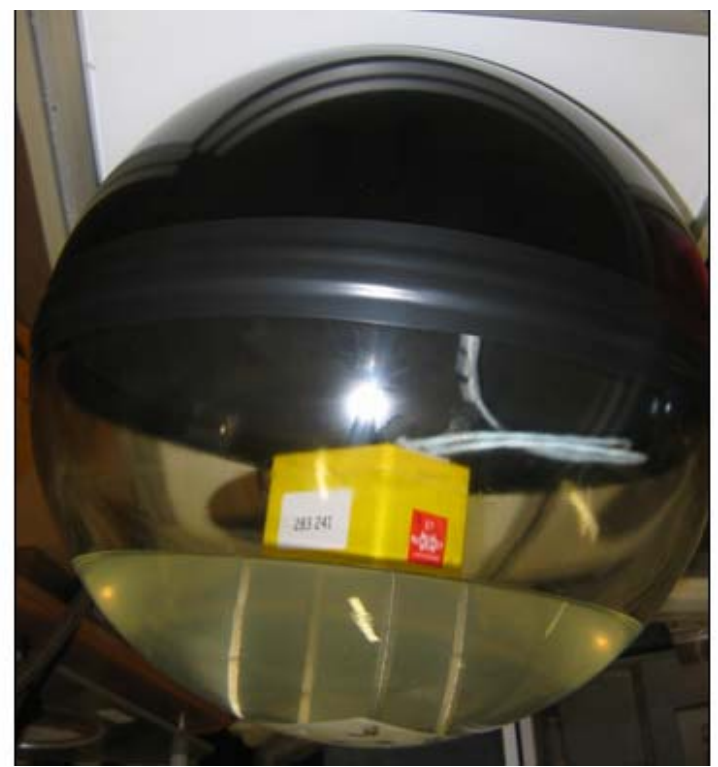

Fig. 7c. TinyTag installation in an optical module sphere.

\subsection{Optical Attenuation Monitoring}

Figure 8 illustrates the fibre-optic interconnections of the full scale mechanical test line. TinyTags are located in storeys 11, 16 and 21 to continuously monitor the optical attenuation (at $\lambda=1550 \mathrm{~nm}$ ) in $\sim 1000$ $\mathrm{m}$ long closed fibre loops, each using two of the seven fibres in one of the three fibre tubes. Each fibre loop has a minimum of $\sim 50$ fusion splices linking the fibres in the various EMC segments. A fourth fibre loop is much longer, around $5.2 \mathrm{~km}$, and links many of the 15 remaining fibres in an undulating path containing several hundred fibre splices.

Figure 9 shows the entry of optical fibres and electrical conductors from the two penetrators into an electronics container. A small printed circuit board serves the power conductors while the optical fibre fusion splices are accommodated in the protective cassettes above. The three stainless steel fibre tubes make transitions into polyurethane tubes within the adhesive potting plug. The polyurethane tubes convey the fibres to the splice cassettes and are intended to protect them from damage during the line assembly process. 


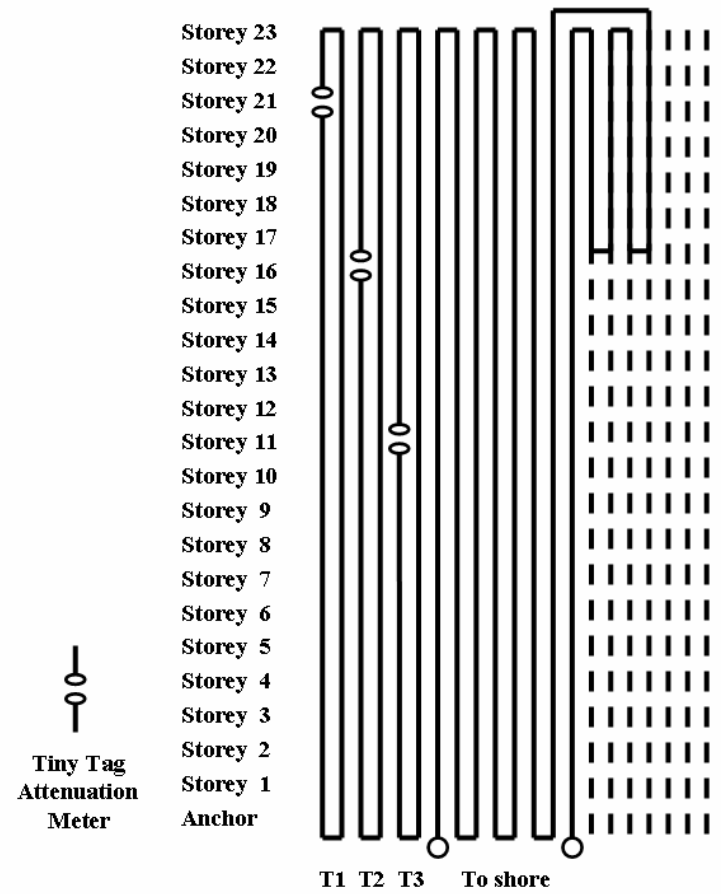

Fig. 8. Interconnections of the optical fibre monitoring loops (bold lines; unused fibres shown in dotted lines).

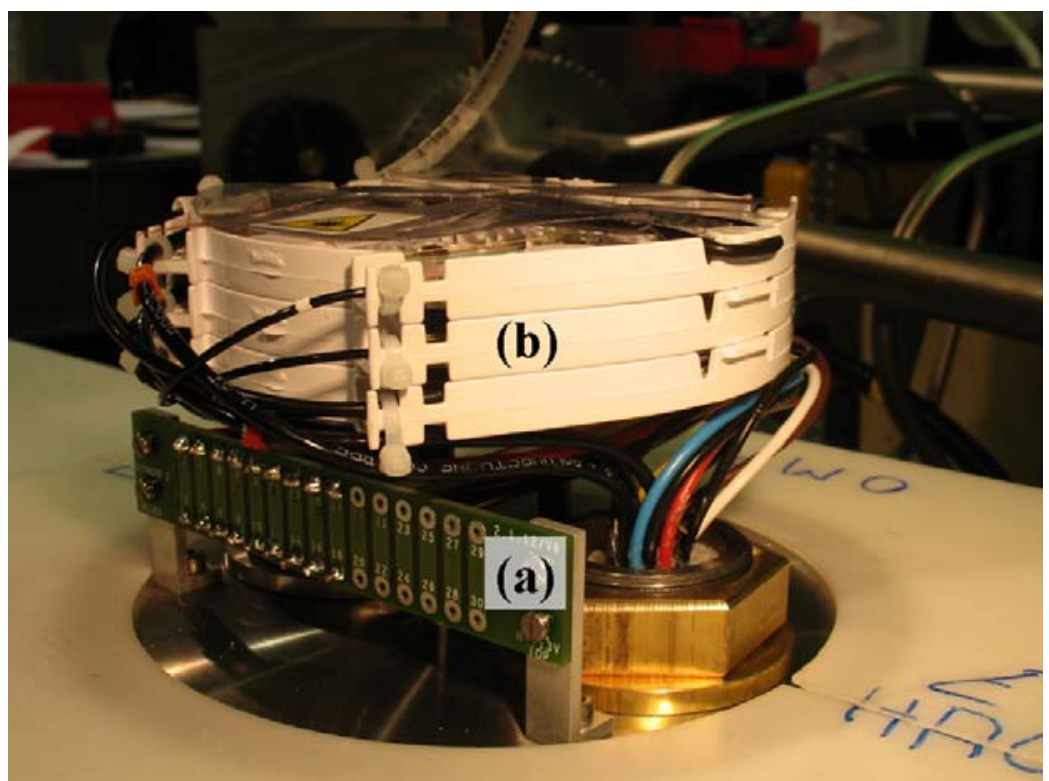

Fig. 9. Detail of the electrical chaining PCB (a) and the protection of fibre-optic splices and pigtails in cassettes (b) mounted on the top flange of an electronics container.

The terminations of the long fibre loop use two of the four fibres in an underwater-mateable electrooptical connector ${ }^{10}$. Following submarine connection ${ }^{11}$ on April 13, 2005, the loop was connected via a $300 \mathrm{~m}$ interconnecting link (IL) cable to the ANTARES deep sea junction box [6], and then to the shore station through the $42 \mathrm{~km}$ main electro-optical undersea cable (MEOC). This permitted real time OTDR ${ }^{12}$

\footnotetext{
${ }^{10}$ Mark II electro-optical connector by Ocean Design Inc, Daytona Beach, Florida 32114, USA,

${ }^{11}$ Using the IFREMER Victor 6000 remotely-operated submarine

${ }^{12}$ Optical Time Domain Reflectometry
} 
monitoring of the attenuation in the long fibre-optic loop, unlike the TinyTags, whose data could only be analyzed following line recovery.

Figure 10a shows the mounting of a TinyTag and an optical attenuation monitor ${ }^{13}$ within an electronics cylinder. Containers with this installation used a third connector to access the RS232 port of the TinyTag monitoring the optical loop. Figure 10b shows the electrical connections of the TinyTag and the effect of applying (uncalibrated) bending stress to a fibre loop, while fig. 10c shows the calibration of the voltage output of the optical attenuation monitor.

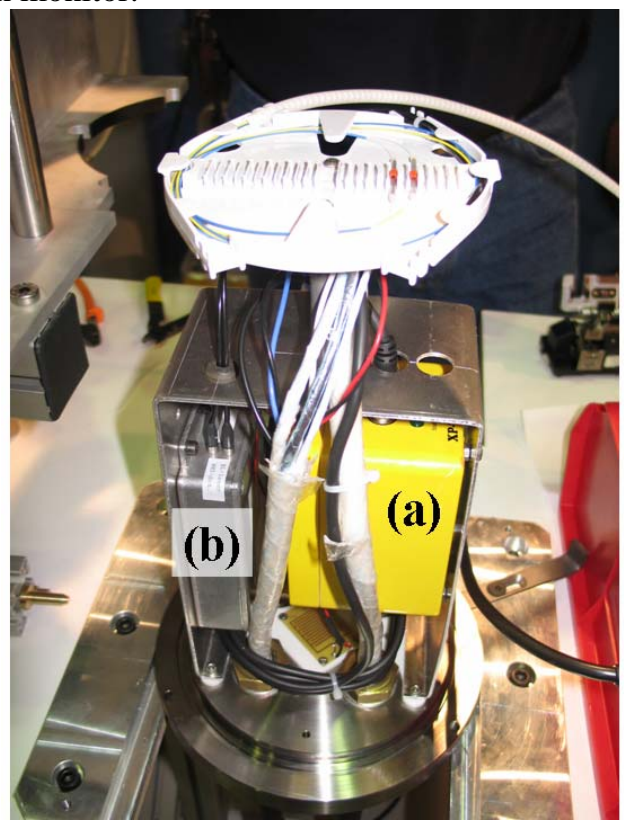

Fig. 10a. Mounting of a TinyTag (a) and optical attenuation monitor (b) in an electronics container.

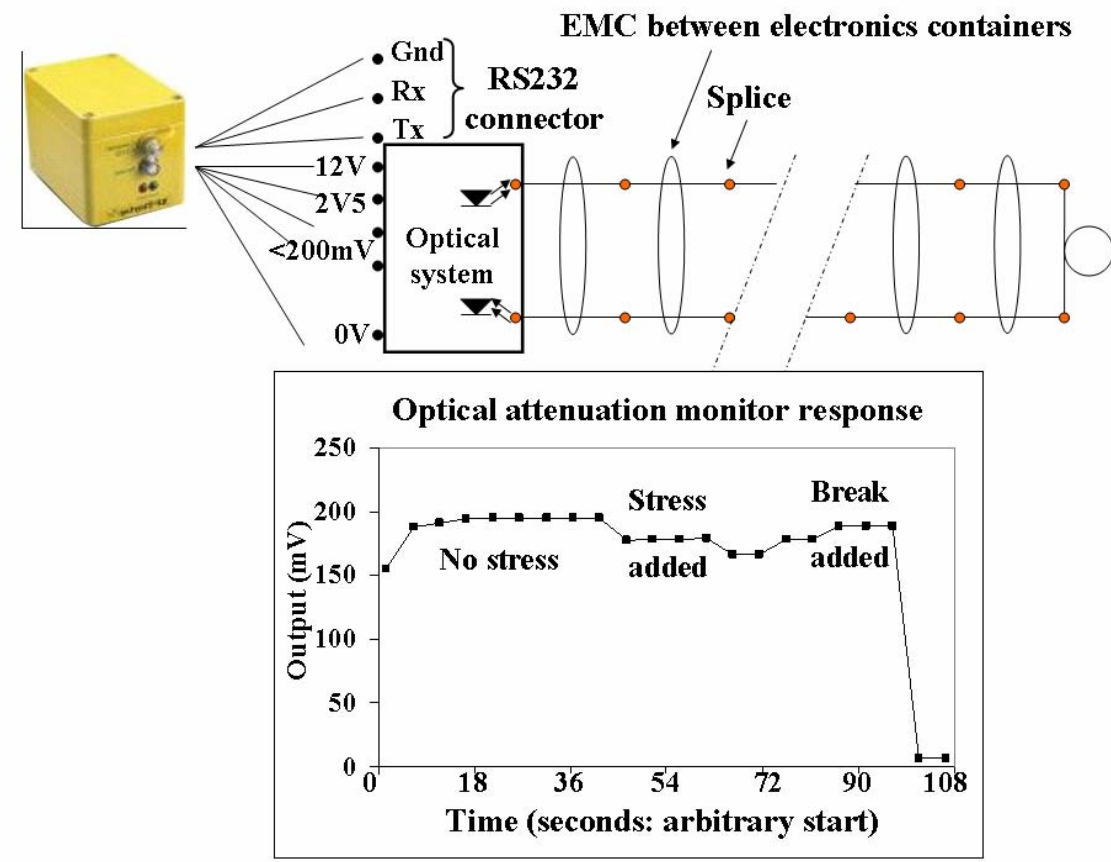

Fig. 10b. TinyTag connection to an optical attenuation monitor and voltage input to TinyTag depending on the condition of the optical fibre.

\footnotetext{
${ }^{13}$ Model 043936, Baas R\&D, 2740AC Waddinxveen, Netherlands
} 


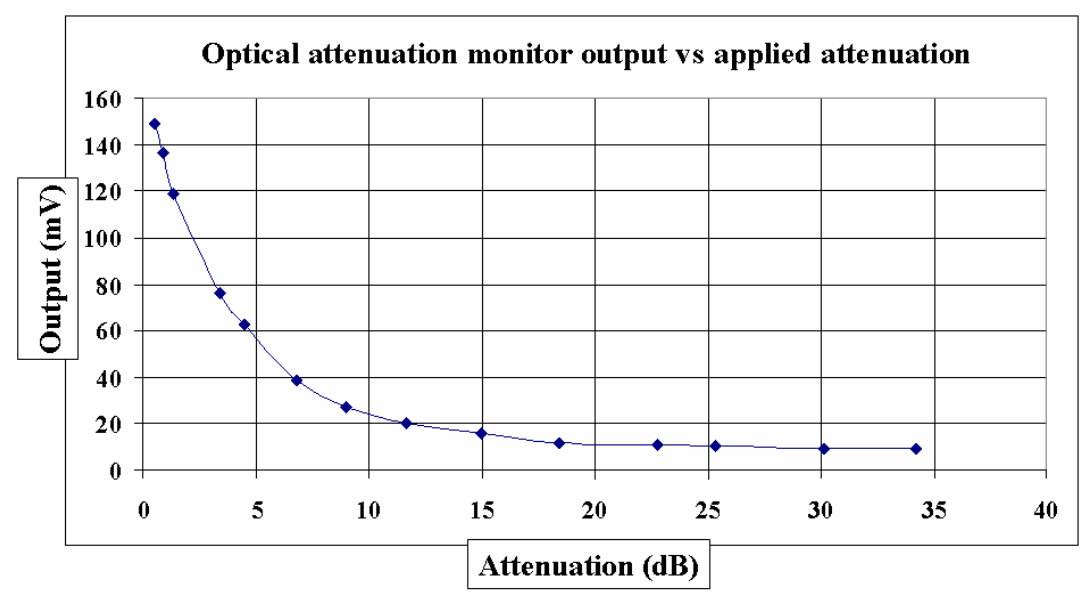

Fig. 10c. Calibration curve for the output voltage of the optical attenuation monitor.

\section{Results from the line deployment}

The full-scale mechanical line was deployed in two phases on March 15 and 17, 2005 in smooth conditions (sea state 1; wind speed $\leq 5$ knots, wave height $\leq 50 \mathrm{~cm}$ ). Immediately prior to immersion, in tests conducted on the ship's deck, the attenuations in the four fibre-optic loops were checked and the attenuation-distance profile in the long loop measured ${ }^{14}$ (fig. 11) using a test cable temporarily plugged into the deep-sea mateable connector. Light was injected in both directions through the open-ended fibre path. Reflection spikes are seen at the unterminated ends of the fibre. The attenuation profiles show a total of $13.8 \mathrm{~dB}$ over the $5.2 \mathrm{~km}$ path length. The data of fig. 11 represent a baseline with which data taken from the shore station were later compared.

In the first phase of deployment on March 15, 2005, the line was lowered to a depth of $2000 \mathrm{~m}$ (anchor datum) where it remained suspended for $\sim 1 \mathrm{hr}$ before being lowered to within $50 \mathrm{~m}$ of the sea floor, where it was suspended for 20 min before being placed on the bottom and the winch cable detached. Around 30 minutes later the acoustic release signal triggered the line ascent, and the buoy reached the surface $\sim 1 \mathrm{hr}$ later. The line spent a total time of around $2^{1 / 2}$ hours at a depth of $2000 \mathrm{~m}$ or greater. The line was recovered onto the deployment ship and the data extracted from the TinyTags monitoring the leak sensors and optical attenuations.

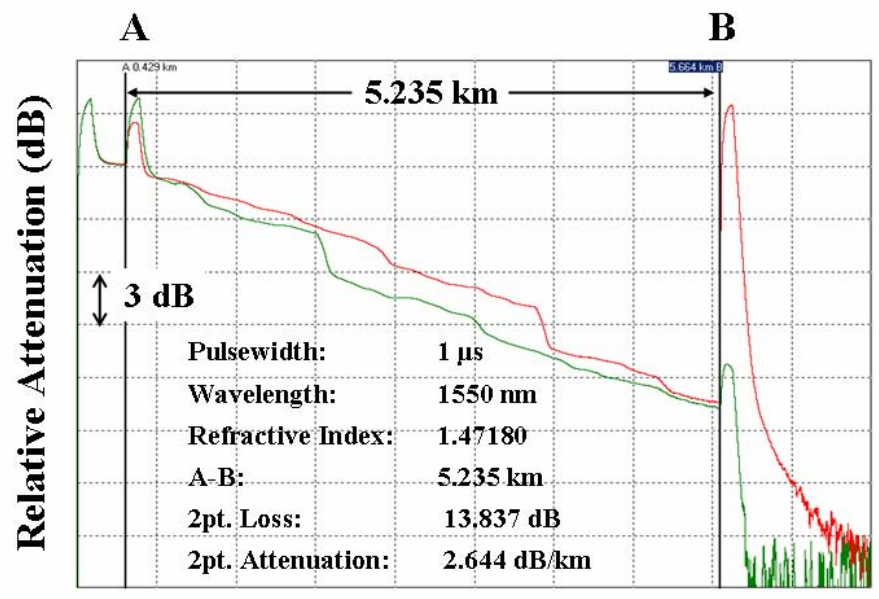

\section{Distance (km)}

Fig. 11. Pre-immersion attenuation-distance profile over the $5.2 \mathrm{~km}$ fibre path. (from ANDO AQ7250 optical time domain reflectometer; the two traces correspond to light injected in opposite directions.)

\footnotetext{
${ }^{14}$ Using ANDO AQ7250 Time domain reflectometer, $\lambda=1550 \mathrm{~nm}$
} 
The time-depth correlation of signals from the optical TinyTags is shown in fig. 12. For each loop the signal loss was no more than $4 \mathrm{~dB}$ during the immersion period and was found to be reversible when the line was recovered. Furthermore, in a deck test immediately following recovery, the attenuation-distance profile of fig. 11 in the long fibre loop was reproduced with the test cable reconnected to the deep-sea mateable connector.

No evidence for leaks was found in any of the 23 leak detectors. Figure 13 shows a typical time profile of the signal from a leak detector over a period starting several days before, and spanning the first immersion phase. The $\mathrm{mV}$-scale fluctuations are due to day/night temperature variations.

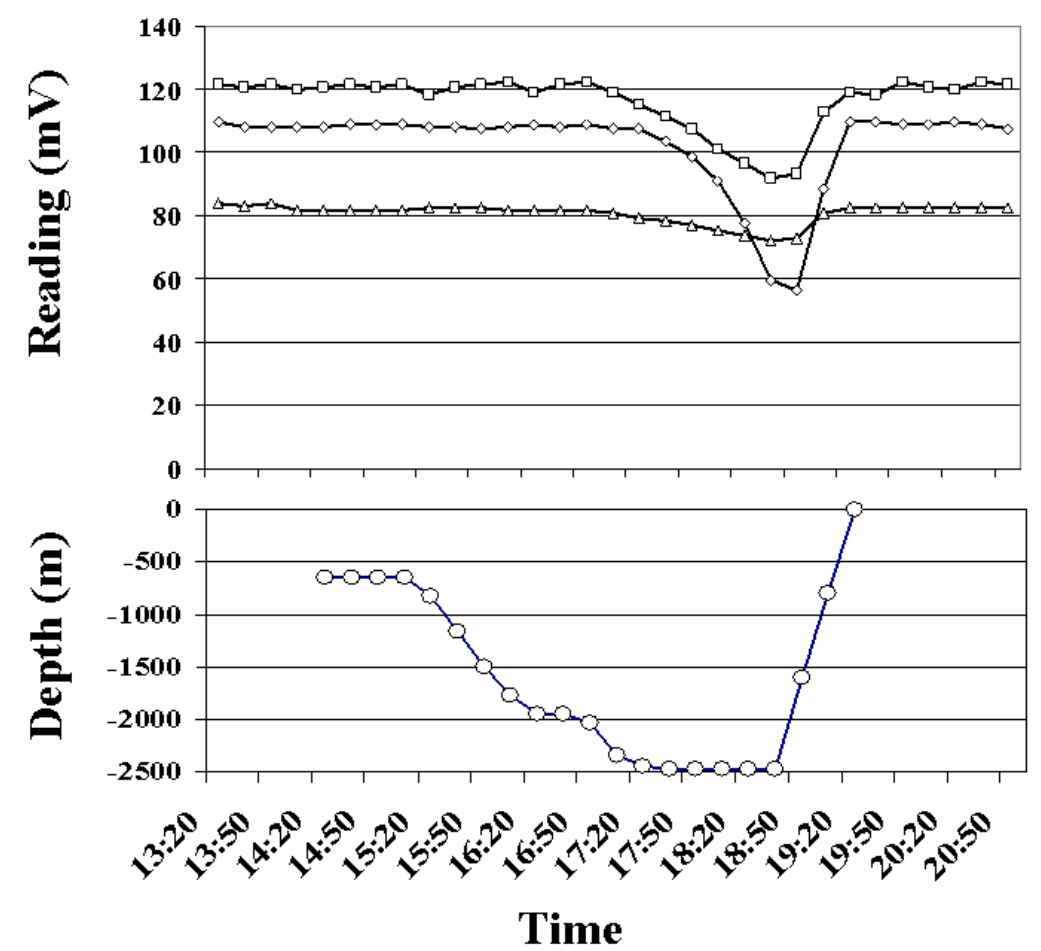

Fig. 12. Time-depth correlation of signals from the optical attenuation sensors positioned in storeys 11 (diamonds), 16 (squares) \& 21 (triangles) during the first phase of line deployment, March 15, 2005.

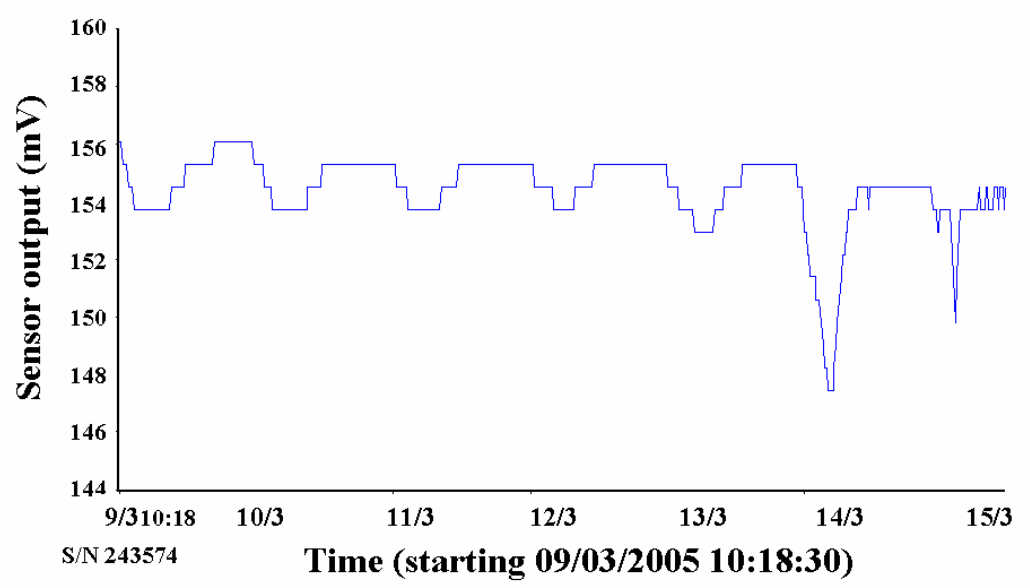

Fig. 13. Signal from storey 12 leak detector for a period before and during the first deployment phase. The spikes on 14/3, 15/3 are due to temperature effects from heating by the sun during the deployment and recovery; a leak would have resulted in a signal level of $\sim 30 \mathrm{mV}$. 
The results from the first immersion phase were considered encouraging enough to proceed with the long-term second phase. The line was re-lowered and deposited on the seabed on March 17, 2005. On April 13 the submarine interlink cable connection was made to the undersea junction box. From this time on, OTDR measurements ${ }^{15}$ of the long fibre-optic loop were possible from the shore station.

Shore-based OTDR data immediately showed that a severe loss in fibre-optic transmission had occurred during or following the second immersion. Figure 14 illustrates the OTDR profile through the MEOC, the undersea junction box, the $300 \mathrm{~m}$ IL cable and into the $5.2 \mathrm{~km}$ fibre path in the test line. The large reflection peaks are seen at the ends of the $42 \mathrm{~km}$ MEOC and the IL cable. Around $336 \mathrm{~m}$ beyond the IL connection to the line, light transmission is almost completely lost at a position corresponding to that of a penetrator linking an EMC segment into an electronics container. Identical behaviour at the same position is seen when light is injected into either end of the fibre path.

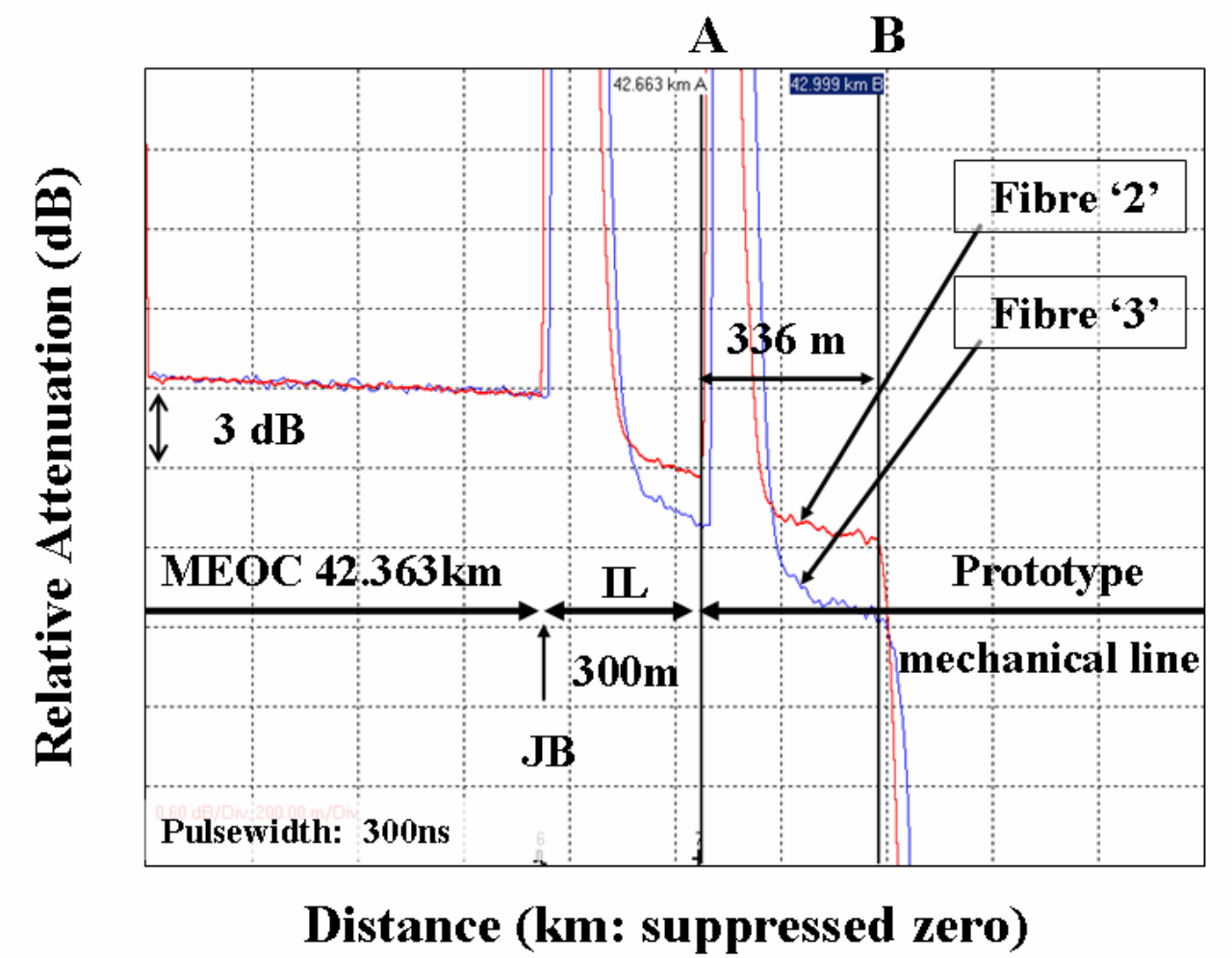

Fig. 14. Post-immersion attenuation-distance profile over the full fibre path from the shore (from ANDO AQ7250 optical time domain reflectometer; the two traces correspond to light injected in opposite directions.)

Following these disappointing results from on-shore OTDR measurements, the line was recovered in mid-May 2005 and re-measured. TinyTag data (fig. 15) showed that transmission in the other three fibre loops had been lost very soon after the second deployment, and that two of the three loops (those read by TinyTags in storeys 11 and 21 with fibre loops in tubes (1) and (3) respectively - fig. 8) did not recover, even partially, after the line was returned to the surface. These measurements are correlated with the data seen in the shore-based OTDR measurements which view the fibre loop whose extremities pass through fibre tubes (1) and (3).

Further OTDR measurements on the recovered line showed additional attenuation peaks around $15 \mathrm{~m}$ beyond the cut-offs seen in fig. 14, an increment corresponding to the inter-storey spacing. Upon partial dismantling of the fibre-optic loops, multiple breaks in fibre-optic continuity could be seen in OTDR profiles which were correlated with the entry points of the EMC segments into the electronics containers. In none of these containers, however, was a water leak detected by the corresponding TinyTag, nor were any traces of water seen.

15 Using ANDO AQ7250 and HP E6000C OTDR instruments 


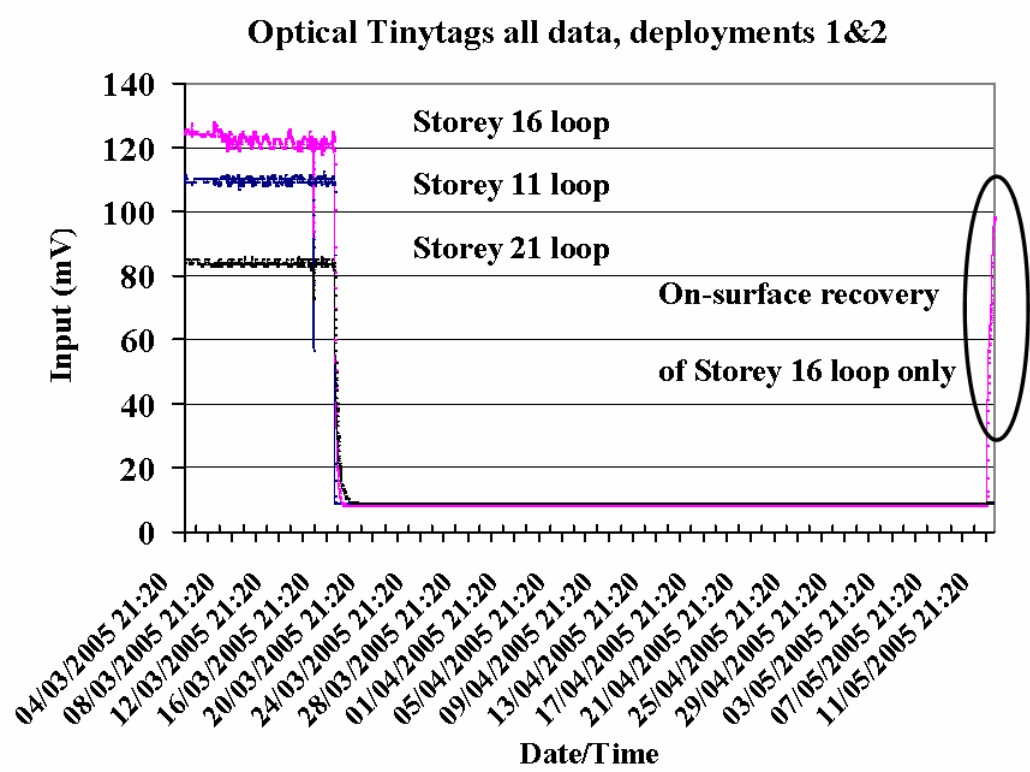

Fig. 15. Time profiles of optical attenuation signals into the three TinyTag data loggers for the whole deployment period.

Figure 16 illustrates the signals from the leak sensors in storeys 1, 12 and 23 (top). The signals on all sensors remain around $150 \mathrm{mV}$, indicating no leaks.

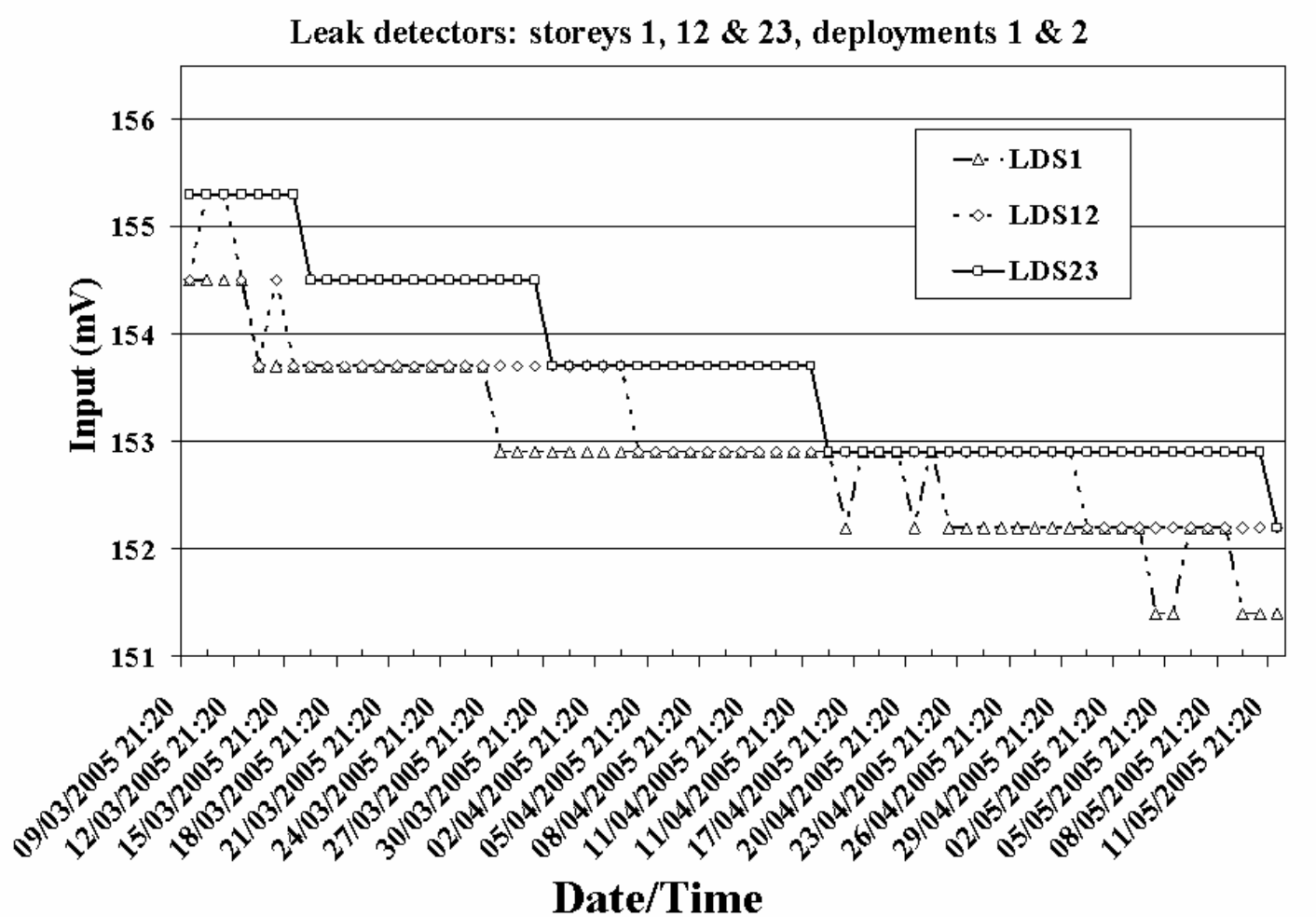

Fig. 16. Signals from leak sensors in containers at the top, middle and bottom of the line over a period before and including the two phase deployment and immersion period. A leak would have resulted in a signal level of $\sim 30 \mathrm{mV}$. 
Following disassembly of the line, visual inspection of the penetrator flanges (fig. 17) showed evidence for adhesive failure of the acrylic potting to the metal surfaces of the fibre tubes and/or the penetrators.

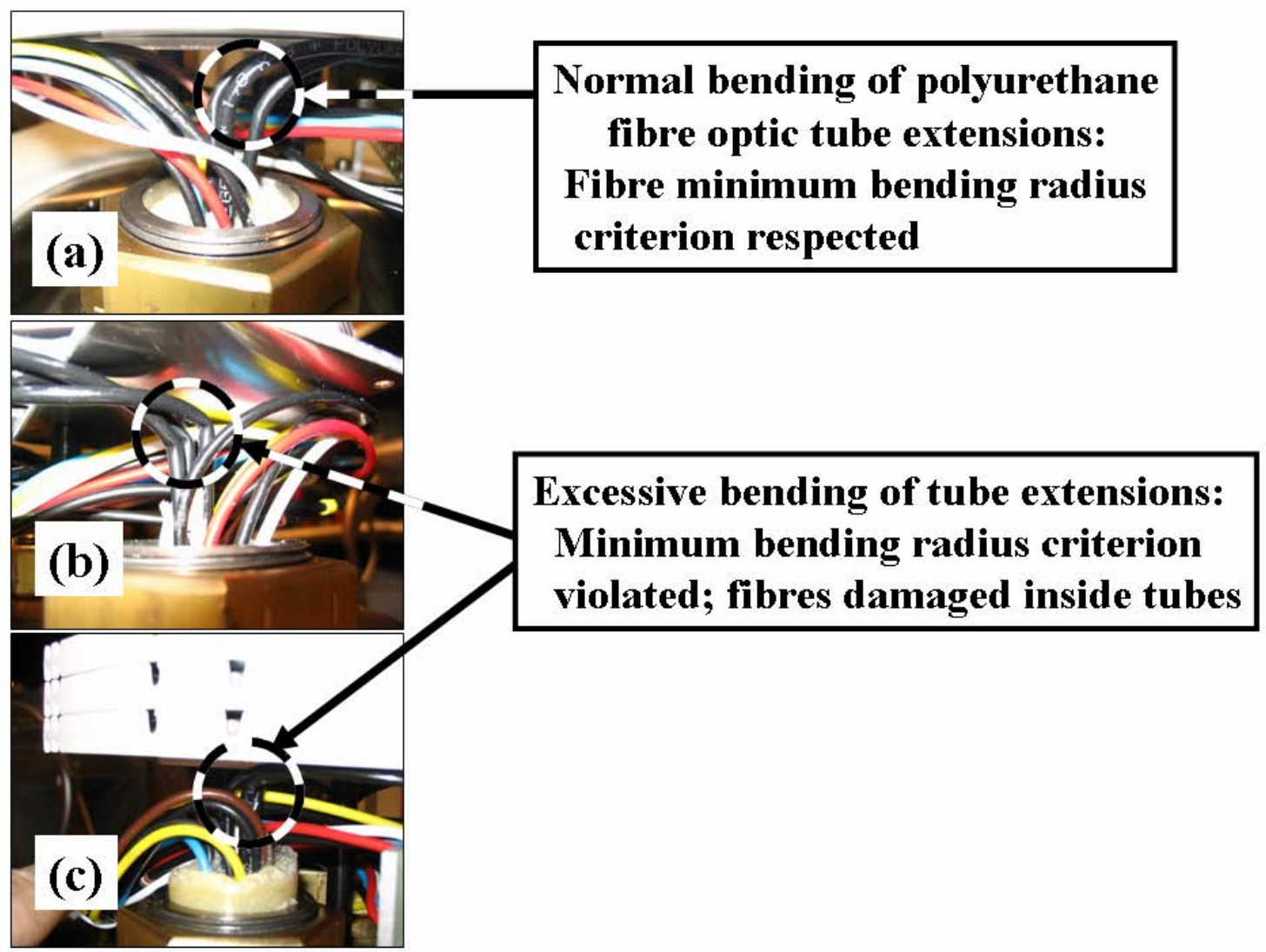

Fig. 17. Illustration of potting adhesive failure in NWB penetrators; (b) slippage of the three fibre tubes through the potting; (c) adhesive failure between the potting plug and the penetrator bore. Undamaged potting is shown for comparison in (a).

These failures occurred under the $\sim 2700 \mathrm{~N}$ shear force on the $11.4 \mathrm{~mm}$ diameter EMC core (fig. 4) generated by the $\sim 250$ bar differential between the cylinder pressure of 1 bar and the maximum sea pressure. Figure 17b illustrates how the fibre tubes have been pushed into the electronics container, impacting the splice cassettes then bending the polyurethane tube extensions and the fibres within, to violate the fibre minimum bend radius criterion ${ }^{16}$, in some cases snapping the fibres. Figure 17c shows an instance of migration of the entire acrylic potting plug into the electronics container through the cylindrical bore of a NWB penetrator. As a result of both kinds of slippage, around $6 \%$ of the optical fibres in the segments of EMC were found to be either broken, or to have an increased attenuation through excessively tight bending, at a point near the penetrators.

\section{The Investigation of mechanical shocks and torsion during deployment}

The full-scale mechanical line was equipped with a variety of autonomous instruments to record acceleration, depth, compass heading and tilt in three axes. Instruments were installed on the deployment ship, at the top storey and base (anchor) of the line and also immediately above the flotation buoy at the end of the $2.5 \mathrm{~km}$ steel winch cable.

\footnotetext{
${ }^{16}$ Following measured attenuations of $0.1 \mathrm{~dB} \& 3 \mathrm{~dB}$ at bending radii of $30 \mathrm{~mm} \& 8 \mathrm{~mm}$ in Corning SMF28 optical fibre at $\lambda=1550$ $\mathrm{nm}$. A minimum bend radius criterion of $30 \mathrm{~mm}$ was therefore respected at all stages during the line construction.
} 
A pair of TempHuRA (“temperature, humidity, rotation, acceleration”) instrument packages (fig. 18) [7] was installed in titanium cylinders at storey 23 and on the line anchor. Each TempHuRA contained a battery-powered PC-based DAQ reading a hygrometer ${ }^{17}$, a PT100 thermometer, a tri-axial accelerometer ${ }^{18}$ and a combined pitch and roll tilt-meter and compass ${ }^{19}$. Accelerometer data were acquired at $200 \mathrm{~Hz}$ and averages taken of 10 successive readings. Other sensor data were acquired at $2 \mathrm{~Hz}$.

The TempHuRA packages were active during the transportation of the line to the deployment ship, the 3-hour sea voyage to the ANTARES site, and during the deployment. During the line transport and deployment phase no shocks with amplitudes exceeding $\pm 1.3 \mathrm{~g}$ were seen on any axis; levels far below the acceptable limits for line mechanical components and the EMC.

During the deployment operation the largest acceleration recorded was at the acoustic release of the deadweight, but this was less than $0.2 \mathrm{~g}$ on any axis, including the vertical.

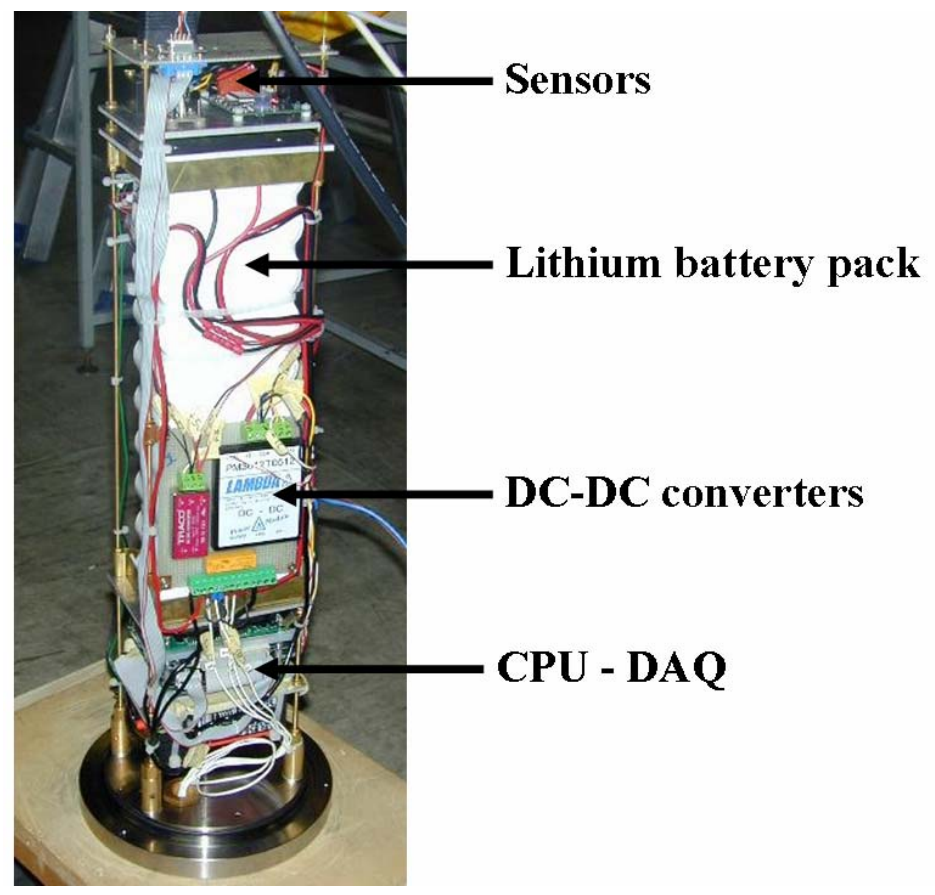

Fig. 18. 'TempHuRA' package incorporating an electronic compass together with three axis tiltmetry and accelerometry.

During the descent, time-heading data from the electronic compasses (fig. 19) indicated that the top of the line rotated 8 times while the base, with the much greater inertia of the anchor, did not. Since the tension of the line was constant during the descent this effect is probably related to the de-spooling of the winch cable and its inherent winding angle. The differential twist of eight rotations between the head and base of the $450 \mathrm{~m}$ long line, equivalent to $\sim 6.4^{\circ}$ per metre, was well within the torque specification of the EMC.

Additional instrumentation allowed the monitoring of winch cable tension and ship motion (heave, pitch and roll). A recording dynamometer ${ }^{20}$ was installed in the cable above the buoy at the top of the detector line. This unit also included a pressure sensor (for turn on at a predetermined depth and for forcedepth profiling) together with vertical and radial accelerometers. Ship motion was recorded using a dynamic motion sensor ${ }^{21}$ installed near the winch cable exit at the stern of the ship.

\footnotetext{
${ }^{17}$ Honeywell model HIH-3605 relative humidity probe

${ }_{18}^{18}$ Model 2422-10: mfr; Silicon Designs Inc, Issaquah, WA 98027, USA

${ }^{19}$ Model TCM2: mfr; PNI Corp., Santa Rosa, CA 95403, USA

${ }^{20}$ Model FD2. Mfr; Sixaxes, 95100 Argenteuil, France

${ }^{21}$ Seatronics Model TSS DMS505: measurement ranges $\pm 10 \mathrm{~m}$ (heave), $\pm 30^{\circ}$ (pitch \& roll)
} 


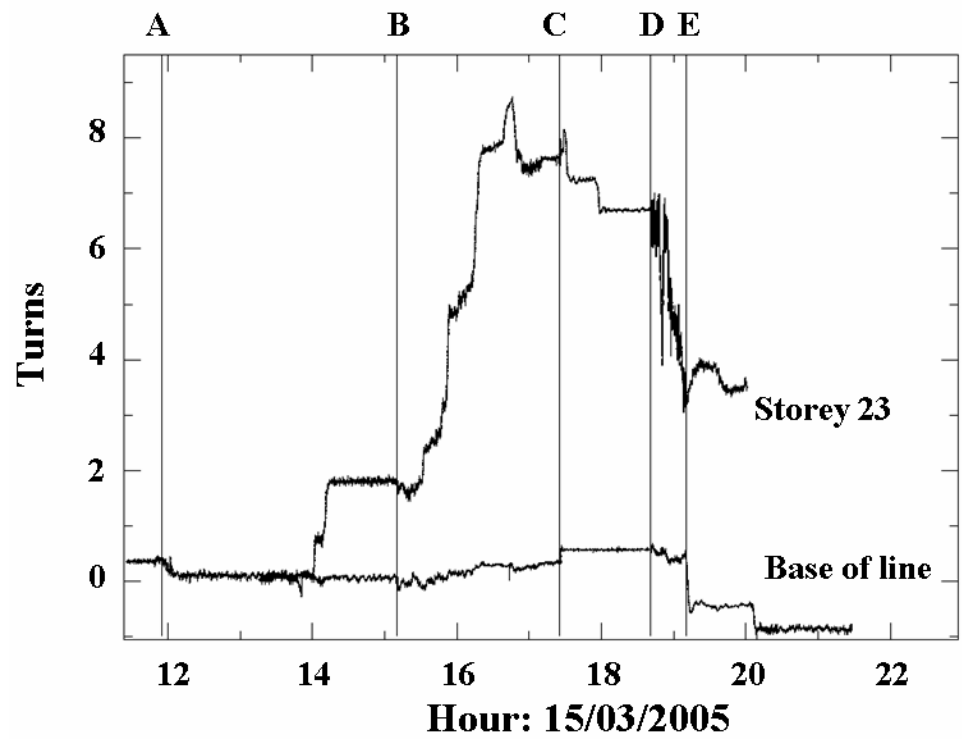

Fig. 19. Logged rotation of the line head (upper trace) and anchor (lower trace) during the first deployment operation: A) anchor at sea surface; B) line fully immersed (anchor at -450 m) \& start of descent; C) first touch on seabed; D) anchor acoustic release triggered; E) line buoy at sea surface.

Surprisingly (fig. 20, [8]), despite the calm sea conditions with recorded heave around $\pm 15 \mathrm{~cm}$ and $\mathrm{pitch} /$ roll $<1^{\circ}$, dynamic tension fluctuations of $\sim \pm 2000 \mathrm{~N}$ were measured (superimposed on the static tension of $7300 \mathrm{~N}$ from the line's own weight) with the winch stopped and the line hanging at constant depth of $2080 \mathrm{~m}$. While these fluctuations presented no problem in this deployment, the data suggested that tension fluctuations in more severe (yet still relatively modest) sea conditions might cause 'negative-g' unloading of the winch cable, with the risk of 'snap-loading' on the next positive-g half cycle which might break the cable or damage a real ANTARES detection line being deployed.

To avoid this, and to assure a much greater safety margin in future deployments, it was decided to lengthen the woven synthetic rope shock absorber between the winch cable and the line flotation buoy from $10 \mathrm{~m}$ to $50 \mathrm{~m}$.
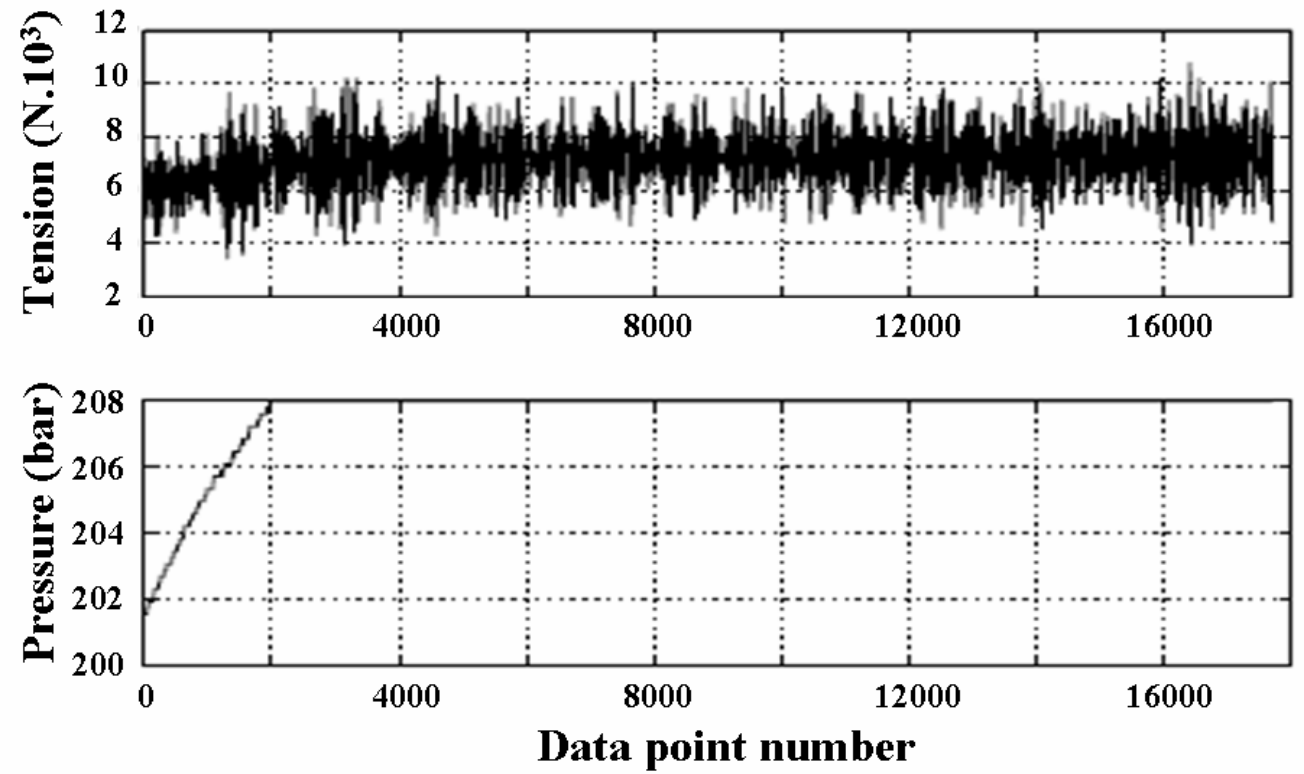

Fig. 20. Correlated data from the winch cable dynamometer and its associated depth gauge, showing tension fluctuations of $\pm 2000 \mathrm{~N}$ centred on the line static weight of $7300 \mathrm{~N}$, under calm sea conditions. 
The conclusion from studies of the various mechanical sensors was that there was no evidence of events capable of inducing a high enough tension, tilt angle or shock acceleration that could be responsible for the observed fibre-optic deterioration. The effect seen was determined therefore to be exclusively due to the shear force on the EMC core cross section caused by the 250 bar pressure differential.

\section{A prototype undersea acoustic detection module}

AMADEUS- $0^{22}$, a prototype autonomous device for the study of the acoustic noise in the deep sea, was installed in a standard titanium electronics container attached to the line anchor. The package (fig. 21) is intended as a precursor for a future feasibility study of acoustic detection of neutrinos with energies exceeding $10^{17} \mathrm{eV}$. Theoretical production models suggest (with large variations) that the flux of such high energy extraterrestrial neutrinos is extremely low; less than one interaction per $\mathrm{km}^{2}$ per year. Very large neutrino telescopes, with instrumented volumes of several tens or hundreds of $\mathrm{km}^{3}$, might be required for their detection.

A neutrino interaction in the target volume would produce a cascade of secondary particles, highly collimated and following the incoming neutrino trajectory. These secondary particles would dump energy into the sea water, leading to an almost instantaneous heating and consequently to a local expansion. The resulting acoustic pressure pulse could be detected by an array of hydrophones to reconstruct the neutrino interaction. Since the acoustic attenuation length in sea water is of the order of km compared to around 50 $\mathrm{m}$ for Cherenkov light, a very large volume could be instrumented at low granularity using hydrophones, making possible a very large volume underwater neutrino telescope which would otherwise be prohibitively expensive using optical detectors.

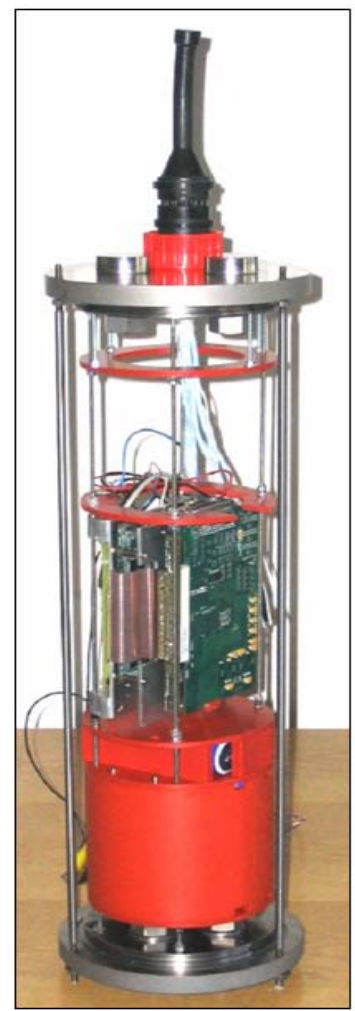

Fig. 21. The AMADEUS-0 package: battery pack at the bottom, with the PC, ADC and timer cards above.

The AMADEUS-0 package contained electronics to amplify, timestamp and store the signals from five piezo-acoustic transducers ${ }^{23}$ attached to the inside of the cylinder using a polyurethane adhesive. Each

${ }^{22}$ AMADEUS-0: “Autonomous Module for Acoustic DEtection Under the Sea”

23 PZT5A ceramic, thickness 10/20 mm, diameter $25 \mathrm{~mm}$ 
channel was equipped with a custom-designed low noise two stage pre-amplifier with a dynamic pressure sensitivity of $\sim 1 \mathrm{~V} / \mathrm{Pa}$. In addition, the signals from one sensor were extracted after the first amplifier stage (sensitivity lower by a factor of $\sim 100$ ), thus facilitating the measurement of large signals without overmodulation. The sensitivity of the sensor/amplifier combination is flat between $3 \mathrm{kHz} \& 60 \mathrm{kHz}$; the frequency range relevant for acoustic particle detection [9]. At lower frequencies a filter removes the expected steeply increasing deep-sea acoustic noise.

The readout electronics and DAQ system were designed for limited space, low power consumption, and autonomous data taking following deployment. Acoustic data were taken at $500 \mathrm{kSamples} / \mathrm{s}$ using a PC-board ${ }^{24}$ and ADC card ${ }^{25}$. Silence was achieved during measurement periods by temporarily storing the data on a 1 GB compact flash card before copying to an 80 GB hard disk during measurement pauses. The package had a total power consumption of $\sim 8.5 \mathrm{~W}$ and was powered from a lithium battery sufficient for autonomous operation of around 20 hours.

Measurements could be started automatically after a predefined time interval adjustable from zero (immediate) to a few months. Following system boot-up, data taking proceeds until the hard disk is full, the batteries are exhausted or the system is stopped manually. The data taking sequence interleaved measurements with a single sensor (full sampling rate of $500 \mathrm{kHz}$ ) with reduced-rate measurements using up to four sensors to look for inter-sensor correlations. The programming of the data taking mode and the readout of data used TCP/IP through a standard ANTARES flange-mounted multi-pin connector.

Data taking proceeded in two phases. In the first phase on March 15, 2005, the DAQ was started by hand before deployment to take data during the descent to the sea bed and the subsequent ascent which took place on the same day. Differences in the noise level at different depths and during different phases of the sea operation were observed.

Figure 22 shows a sample spectrogram (frequency spectrum vs. time) obtained for a two-second period of data taking. The signals of an operating acoustic transponder (receiving at $8 \mathrm{kHz}$ and re-emitting at 12 $\mathrm{kHz}$ ) used for underwater acoustic positioning in the ANTARES telescope are highlighted at the lower centre of the spectrogram, demonstrating the ability of the system to separate the frequencies of acoustic signals in the deep sea.

On March 17, 2005 the line was redeployed and remained on the sea bed for almost two months. The second AMADEUS-0 measurement phase was started by timer delay on April $15^{\text {th }}$. Data were taken for several hours. The analysis of these data will be published in a dedicated work.

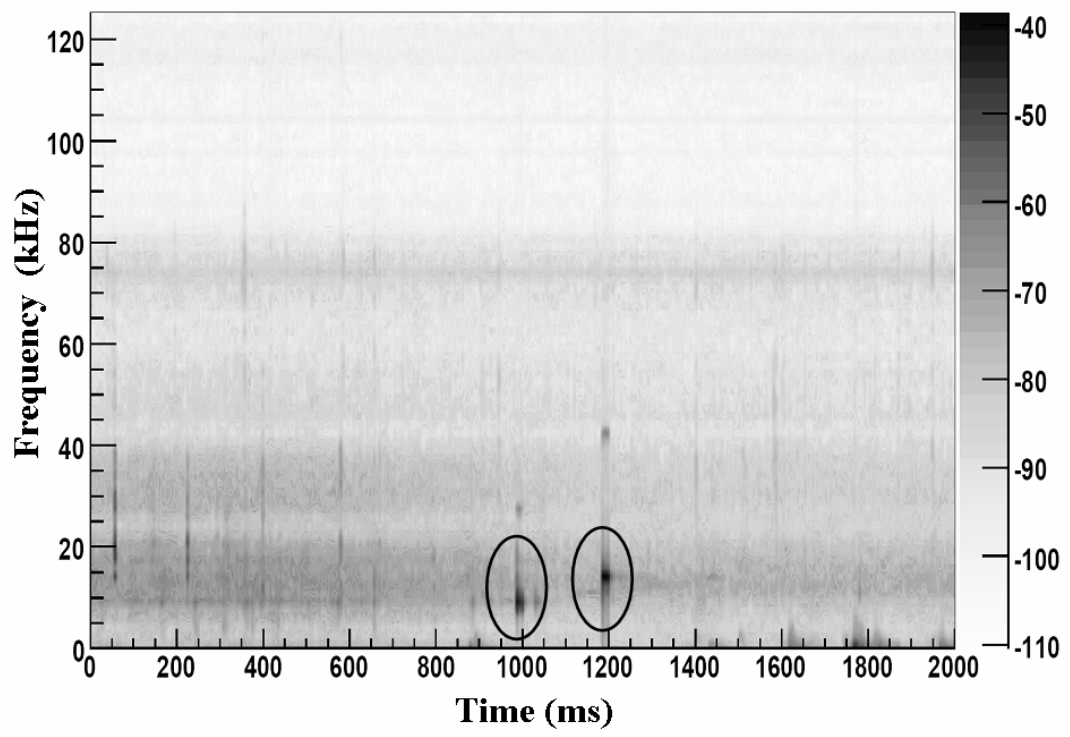

Fig. 22 Spectrogram for a $2 \mathrm{~s}$ measuring interval (abscissa). The left vertical axis indicates the frequency in $\mathrm{kHz}$. The gradation gives the power spectral density in $\mathrm{dBV} / \sqrt{\mathrm{Hz}}$.

${ }^{24}$ Soekris net4801 running Debian Linux

25 Meilhaus ME-4650, 500 kSamples/s, 16 bit resolution, input range $\pm 10 \mathrm{~V}$ 


\section{Conclusions from the line deployment and resulting electromechanical cable improvements}

The principle objective of the ANTARES full scale mechanical test line was the study of leak-tightness of the proposed electronics container scheme and the behaviour of the proposed design for the electromechanical cable. The line also served as a deployment platform for a prototype acoustic detection system which successfully separated and recorded deep sea acoustic signals in the 5-20 kHz frequency band. Such a system might one day find application in the acoustic detection of neutrinos with energies exceeding $10^{17} \mathrm{eV}$.

The two-stage deployment of the line confirmed the sealing approach chosen for the electronics containers and also successfully revealed a significant weakness in the design of the penetrator terminations of the inter-storey EMC segments. These problems were related to the poor adhesion of the acrylic resin potting to the stainless steel fibre tubes and titanium penetrator bore under the shear force generated by the pressure differential between the container internal pressure of 1 bar and the maximum sea pressure of 250 bar.

A series of remedies has been put in place. A systematic study of cable creepage was instigated using pressure vessels in France and Denmark, and an investigation of adhesive properties of a variety of structural epoxy and adhesive resins was undertaken.

The adhesive has been changed to a two-part epoxy ${ }^{26}$ with better adhesion to metal surfaces, while the inner extremity of the EMC penetrator bore has been equipped with a threaded insert that serves as a block to the migration of the potting plug into the electronics chamber. In addition, each fibre tube is terminated in a ribbed ferrule which is embedded in the adhesive, the ribbing grooves blocking any movement of the tube.

Results from compression tests on prototype EMC penetrator terminations in the new geometry are very encouraging: no increase of fibre attenuation was seen during the compression/decompression cycle and no movements of the fibre tubes or potting plugs were observed. New cables in the new configuration have been ordered. The first detection line to be equipped with the new cable was deployed in September 2006. As of January 2007, four such lines are in operation in the deep sea. Future publications will deal with the development of the ANTARES electromechanical cable and the final configuration employed.

\section{Acknowledgements}

The authors acknowledge the financial support of the funding agencies. In particular; Centre National de la Recherche Scientifique (CNRS), Commissariat à l'Énergie Atomique (CEA), Commission Européenne (FEDER fund), Région Alsace (contrat CPER), Région Provence-Alpes-Côte d’Azur, Département du Var and Ville de La Seyne-sur-mer, in France; Bundesministerium für Bildung und Forschung (BBF), in Germany; Instituto Nazionale di Fisica Nucleare (INFN), in Italy; de stichting voor Fundamenteel Onderzoek der Materie (FOM) and the Nederlandse organisatie voor Wetenschappelijk Onderzoek (NOW), in the Netherlands; National Authority for Scientific Research (ANCS) in Romania; Russian Foundation for Basic Research (RFBR) in Russia; Ministerio de Educación y Ciencia (MEC), in Spain.

\section{References}

[1] E. Aslanides et al, ANTARES collaboration; The ANTARES proposal; CPPM-P-1999-02, DAPNIA 99-01, IFIC/99-42, SHEFHEP/99-06, Astro-ph/9907432 ANTARES technical design report; http://antares.in2p3.fr

[2] V. Bertin on behalf the ANTARES Collaboration, "Status of the ANTARES neutrino detector", $8^{\text {th }}$ International Workshop on Topics in Astroparticle and Underground Physics/" (TAUP 2003), Nucl. Phys. B Supp. 138 195-197, 2005

[3] P. Amram et al., ANTARES Collaboration, Nucl. Inst. Meth. A484 369-383, (2002) (astro-ph/0112172).

[4] J.A. Aguilar et al., ANTARES Collaboration, Nucl. Inst. Meth. A555 132-141, (2005) (astro-ph/0510031).

[5] M. Ageron et al., ANTARES Collaboration, Nucl. Inst. Meth. A578 498-509, (2007) (astro-ph/0703335)

[6] M. Anghinolfi et al, Nucl. Inst. Meth.A567, 527-530 (2006)

[7] M. Battaglieri et al 'The description of the TempHuRA meter'; INFN/TC-02-15, June 5, 2002

[8]G. Damy; IFREMER DCB/ERT/H0, June 15, 2005; 'Analysis of data from the ANTARES line deployment experiment'

[9] "Study of piezo based sensors for acoustic particle detection”; G. Anton et al. Astropart. Phys. 26: 301-309 (2006)

${ }^{26}$ Araldite 2011, Mfr; Huntsman, B-3078 Everberg, Belgium 